\title{
A wAlbB Wolbachia transinfection displays stable phenotypic effects across divergent Aedes aegypti mosquito backgrounds
}

Perran A. Ross* ${ }^{*}$, Xinyue Gu* $\ddagger$, Katie L. Robinson*, Qiong Yang*, Ellen Cottingham* ${ }^{1}$, Yifan Zhang*, Heng Lin Yeap ${ }^{+*}$, Xuefen Xu*, Nancy M. Endersby-Harshman* and Ary A. Hoffmann*

* Pest and Environmental Adaptation Research Group, Bio21 Institute and the School of BioSciences, the University of Melbourne, Parkville, VIC, Australia

+ CSIRO Land and Water, Black Mountain, Canberra, ACT, Australia

$\ddagger$ These authors contributed equally to this research

${ }^{1}$ Present address: Faculty of Veterinary and Agricultural Sciences, the University of Melbourne, Parkville, Victoria 3052, Australia 
bioRxiv preprint doi: https://doi org/10.1101/2021.06.25.450002; this version posted June 26, 2021. The copyright holder for this preprint (which was not certified by peer review) is the author/funder, who has granted bioRxiv a license to display the preprint in perpetuity. It is made available under aCC-BY-NC 4.0 International license.

Short title: wAlbB and mosquito background

Keywords: Wolbachia, wAlbB, Aedes aegypti, cytonuclear interactions, genomics, population replacement, population suppression

Corresponding author: Perran A. Ross, Bio21 Institute, 30 Flemington Road Parkville, Victoria 3052, Australia, +61415867674, perran.ross@unimelb.edu.au 


\begin{abstract}
Aedes mosquitoes harboring intracellular Wolbachia bacteria are being released in arbovirus and mosquito control programs. With releases taking place around the world, understanding the contribution of host variation to Wolbachia phenotype is crucial. We generated a Wolbachia transinfection $\left(w A l b B^{Q}\right)$ in Aedes aegypti and performed backcrossing to introduce the infection into Australian or Malaysian nuclear backgrounds. Whole Wolbachia genome sequencing shows that the $w A l b B^{Q}$ transinfection is near-identical to the reference $w A l b B$ genome, suggesting few changes since the infection was first introduced to Ae. aegypti over 15 years ago. However, these sequences were distinct from other available wAlbB genome sequences, highlighting the potential diversity of wAlbB in natural Ae. albopictus populations. Phenotypic comparisons demonstrate effects of wAlbB infection on egg hatch and nuclear background on fecundity and body size, but no interactions between wAlbB infection and nuclear background for any trait. The wAlbB infection was stable at high temperatures and showed perfect maternal transmission and cytoplasmic incompatibility regardless of host background. Our results demonstrate the stability of wAlbB across host backgrounds and point to its long-term effectiveness for controlling arbovirus transmission and mosquito populations.
\end{abstract}

\title{
Introduction
}

Wolbachia are intracellular, maternally-inherited bacteria found within about half of all insect species (WEINERT et al. 2015; SAZAMA et al. 2019). Wolbachia-infected Aedes aegypti mosquitoes are being released into field populations as a way of controlling mosquito populations and arbovirus transmission (Ross et al. 2019c). Release programs involve strains of Wolbachia that have been introduced artificially into Ae. aegypti, which do not harbor Wolbachia naturally (GLORIA-SORIA et al. 2018; Ross et al. 2020b). Most Wolbachia infections in mosquitoes induce cytoplasmic incompatibility, where Wolbachia-infected males cannot produce viable offspring with females that are not infected (XI et al. 2005b; MCMENIMAN et al. 2009; WALKER et al. 2011). Wolbachia infections can also suppress virus replication within the mosquito, limiting their ability to transmit dengue, Zika and other arboviruses (TERRADAS AND MCGRAW 2017). Wolbachia can spread through target mosquito populations by inducing cytoplasmic incompatibility, which provides a frequency-dependent fitness advantage to Wolbachia-infected females (HoFFMANN et al. 2011). Wolbachia infections can also be used to suppress mosquito populations through cytoplasmic incompatibility (MAINs et al. 2019; ZHENG et al. 2019; CRAWFORD et al. 2020) as well as deleterious host fitness effects (RITCHIE et al. 2015).

A variety of Wolbachia strains originating from Drosophila and mosquitoes have been introduced into Ae. aegypti through microinjection (FRASER et al. 2017; ANT et al. 2018). These strains show diverse effects on host fitness, virus blocking and cytoplasmic incompatibility (Ross et al. 2019c). Population replacement releases currently involve two Wolbachia strains: wMel and wAlbB. Both strains have successfully established in natural Ae. aegypti populations (HOFFMANN et al. 2011; GARCIA et al. 2019; NAZNI et al. 2019; TANTOWIJOYO et al. 2020) and have similar viral blocking effects (ANT et al. 2018; FLORES et al. 2020). Population replacement programs have demonstrated efficacy against dengue transmission, with fewer dengue cases in areas where Wolbachia infections are at a high frequency in 
the Ae. aegypti population (O'NEILL et al. 2018; NAZNI et al. 2019; RYAN et al. 2019; INDRIANI et al. 2020; PINTO et al. 2021). However, establishment success can vary dramatically in different locations, even with the same Wolbachia strain. For instance, wMel readily invaded Australian Ae. aegypti populations (HOFFMANN et al. 2011; SCHMIDT et al. 2017; O'NEILL et al. 2018; RYAN et al. 2019) but did not reach high frequencies in many parts of Niterói and Rio de Janeiro in Brazil despite multiple rounds of releases (GESTO et al. 2021; PINTO et al. 2021).

Wolbachia release success depends on the properties of the Wolbachia strain, including host fitness costs, cytoplasmic incompatibility, maternal transmission, virus blocking and environmental stability. The wAlbB Wolbachia strain occurs naturally in Ae. albopictus and was the first strain transferred to Ae. aegypti through microinjection ( $\mathrm{XI}$ et al. 2005b). wAlbB has limited effects on dengue virus transmission in its native host (LU et al. 2012; MoussON et al. 2012) but shows strong blocking against a range of arboviruses in Ae. aegypti (BIAN et al. 2010; JOUBERT et al. 2016; ANT et al. 2018; BHATTACHARYA et al. 2020) and complete cytoplasmic incompatibility (XI et al. 2005b). wAlbB infection induces substantial host fitness costs, including reduced tolerance to starvation (Ross et al. 2016; Foo et al. 2019) and thermal stress (ROss et al. 2019b), decreased quiescent egg viability (AXFORD et al. 2016; JOUBERT et al. 2016) and high rates of female infertility following egg storage (LAU et al. 2021). However, wAlbB is relatively stable at high temperatures compared to wMel (Ross et al. 2017b), prompting its deployment in field trials in Kuala Lumpur, Malaysia. Releases of wAlbB led to stable population replacement in some trial locations with a corresponding reduction in dengue transmission (NAZNI et al. 2019). Aedes aegypti carrying $w A \mathrm{AlbB}$ have also been released in population suppression programs that rely on cytoplasmic incompatibility to reduce wild female fertility (MAINS et al. 2019; CRAWFORD et al. 2020).

When a Wolbachia strain is released into a new environment, it is important to understand factors that can affect its establishment. Wolbachia releases are taking place in genetically divergent mosquito populations (GLORIA-SORIA et al. 2016; SCHMIDT et al. 2020) and host genotype may influence release outcomes. Host genotype can influence Wolbachia's effects on host fitness (REYNOLDS et al. 2003; DEAN 2006; KYRITSIS et al. 2019; CARVALHO et al. 2020), cytoplasmic incompatibility (REYNOLDS AND HOFFMANN 2002; BORDENSTEIN et al. 2003) and viral blocking (TERRADAS et al. 2017; FORD et al. 2019). Wolbachia releases in new locations often involve backcrossing to introduce the target Wolbachia infection into locally-adapted mosquitoes (DUTRA et al. 2015; GARCIA et al. 2019; TANTOWIJOYO et al. 2020). Since Wolbachia and mitochondria are co-inherited, Wolbachia infections carry along their associated mitochondria when they invade (RASGON et al. 2006; YEAP et al. 2016), resulting in mosquito populations with foreign mitochondria (GARCIA et al. 2019; TANTOWIJOYO et al. 2020). Mismatches between mitochondrial and nuclear genotypes in other insects can have severe deleterious effects (HOEKSTRA et al. 2013; MEIKLEJOHN et al. 2013; RANK et al. 2020). However, such effects have not been tested in mosquitoes.

In this study, we generated a new wAlbB transinfection in Ae. aegypti with an Australian mitochondrial haplotype and nuclear background, denoted $W \mathrm{AlbB}^{Q}$. We then used antibiotic curing and backcrossing to generate populations to investigate effects of wAlbB infection across two nuclear backgrounds. We found that phenotypic effects were stable across backgrounds, with no evidence for deleterious effects when mitochondrial haplotypes and nuclear backgrounds are mismatched. Whole Wolbachia genome 
sequencing of the $\mathrm{WAlbB}^{\mathrm{Q}}$ transinfection revealed very few changes compared to the reference genome. Our results indicate that wAlbB infections may remain stable in their effects in divergent mosquito populations and across time.

\section{Methods}

\section{Strain production}

\section{Mosquito strains and colony maintenance}

Aedes aegypti mosquitoes were reared in temperature-controlled insectaries at $26 \pm 1{ }^{\circ} \mathrm{C}$ with a $12 \mathrm{hr}$ photoperiod according to Ross et al. (2017a). All populations were maintained at a census size of 400 individuals in BugDorm- 4F2222 (13.8 L) or BugDorm-1 (27 L) cages (Megaview Science C., Ltd., Taichung, Taiwan). Larvae were reared in trays filled with $4 \mathrm{~L}$ of reverse osmosis (RO) water and provided with fish food (Hikari tropical sinking wafers, Kyorin food, Himeji, Japan) ad libitum throughout their development. Embryonic microinjection experiments were performed in a general insectary, where female mosquitoes (5-7 d old, starved for $24 \mathrm{hr}$ ) were fed on the forearm of a human volunteer. Blood feeding of female mosquitoes on human volunteers was approved by the University of Melbourne Human Ethics Committee (approval 0723847). All adult subjects provided informed written consent (no children were involved). All following experiments were conducted in a quarantine insectary; female mosquitoes were fed human blood via Hemotek $®$ membrane feeders (Hemotek Ltd, Blackburn Lancashire, Great Britain) according to PARIS et al. (2018). Human blood was sourced from the Red Cross (Agreement \#16-10VIC-02) and refreshed monthly.

Several Wolbachia-infected and uninfected populations were used in this study (Table 1). The wAlbB infection originated from Ae. albopictus collected in Texas, USA (SINKINS et al. 1995). wAlbB was introduced to Ae. aegypti through embryonic microinjection (XI et al. 2005b) and repeatedly backcrossed to an Australian nuclear background (AXFORD et al. 2016). We then introduced wAlbB into Ae. aegypti with an Australian mitochondrial haplotype and nuclear background (QL) through embryonic microinjection (see below). We have named this transinfection wAlbB ${ }^{\complement}$ based on its mtDNA of Queensland origin.

Table 1. Summary of Aedes aegypti populations used in this study.

\begin{tabular}{|l|l|l|l|l|l|l|}
\hline Name & Origin & $\begin{array}{l}\text { Wolbachia } \\
\text { infection }\end{array}$ & $\begin{array}{l}\text { Mitocho } \\
\text { ndrial } \\
\text { haplotyp } \\
\text { e (see } \\
\text { Table } \\
\text { S2) }\end{array}$ & $\begin{array}{l}\text { Nuclear } \\
\text { background } \\
\text { (estimated \%) }\end{array}$ & $\begin{array}{l}\text { Replicat } \\
\text { e } \\
\text { populati } \\
\text { ons }\end{array}$ & $\begin{array}{l}\text { Experiments } \\
\text { included in }\end{array}$ \\
\hline wAlbB Au & This study & wAlbB & 1 & $\begin{array}{l}\text { Australian - Au } \\
(100 \%)\end{array}$ & 2 & $\begin{array}{l}\text { All phenotypic } \\
\text { assays, genomics }\end{array}$ \\
\hline
\end{tabular}




\begin{tabular}{|c|c|c|c|c|c|c|}
\hline wAlbB My & This study & $w \mathrm{AlbB}^{Q}$ & 1 & $\begin{array}{l}\text { Malaysian - My } \\
(\sim 87.5 \%), \\
\text { Australian - Au } \\
(\sim 12.5 \%)\end{array}$ & 2 & $\begin{array}{l}\text { All phenotypic } \\
\text { assays }\end{array}$ \\
\hline $\begin{array}{l}\text { Uninfected } \\
\mathrm{Au}\end{array}$ & This study & $\begin{array}{l}\text { Uninfected } \\
\text { (tetracycline- } \\
\text { cured) }\end{array}$ & 1 & $\begin{array}{l}\text { Australian - Au } \\
(100 \%)\end{array}$ & 2 & $\begin{array}{l}\text { All phenotypic } \\
\text { assays }\end{array}$ \\
\hline $\begin{array}{l}\text { Uninfected } \\
\text { My }\end{array}$ & This study & $\begin{array}{l}\text { Uninfected } \\
\text { (tetracycline- } \\
\text { cured) }\end{array}$ & 1 & $\begin{array}{l}\text { Malaysian - My } \\
(\sim 87.5 \%), \\
\text { Australian - Au } \\
(\sim 12.5 \%)\end{array}$ & 2 & $\begin{array}{l}\text { All phenotypic } \\
\text { assays }\end{array}$ \\
\hline wAlbB US & $\begin{array}{l}\text { XI et al. }(2005 b) ; \\
\text { AXFORD et al. } \\
(2016)\end{array}$ & wAlbB & 3 & $\begin{array}{l}\text { Australian - Au } \\
(\sim 100 \%)\end{array}$ & 1 & $\begin{array}{l}\text { Microinjection } \\
\text { only }\end{array}$ \\
\hline QL & $\begin{array}{l}\text { Collected from } \\
\text { Cairns, Australia } \\
\text { in } 2018\end{array}$ & $\begin{array}{l}\text { Uninfected } \\
\text { (native) }\end{array}$ & $1,4,5$ & $\begin{array}{l}\text { Australian - Au } \\
(100 \%)\end{array}$ & 1 & $\begin{array}{l}\text { Microinjection } \\
\text { and backcrossing } \\
\text { only }\end{array}$ \\
\hline MY & ANT et al. (2018) & $\begin{array}{l}\text { Uninfected } \\
\text { (native) }\end{array}$ & 2 & $\begin{array}{l}\text { Malaysian - My } \\
(100 \%)\end{array}$ & 1 & $\begin{array}{l}\text { Backcrossing } \\
\text { only }\end{array}$ \\
\hline wMel & $\begin{array}{l}\text { WALKER } \text { et al. } \\
\text { (2011) }\end{array}$ & $w \mathrm{Mel}$ & 1 & $\begin{array}{l}\text { Australian - Au } \\
(100 \%)\end{array}$ & 1 & Heat stress only \\
\hline
\end{tabular}

\section{Embryonic microinjection}

To generate wAlbB-infected Ae. aegypti with an Australian mitochondrial haplotype ( AlbB $^{\complement}$ ), we transferred wAlbB from a donor population (XI et al. 2005b; AXFORD et al. 2016) into uninfected Australian Ae. aegypti (QL). We used two approaches to obtain Wolbachia from the donor population. In the first approach, approximately 10 pairs of ovaries were dissected from females blood fed 4-5 d earlier and crushed gently with a pestle in SPG buffer in a $1.7 \mathrm{~mL}$ Eppendorf tube, then processed according to XI AND DOBSON (2005). In the second approach, cytoplasm was removed from donor eggs and injected directly into the recipient embryo (XI et al. 2005a).

For all experiments, recipient eggs were collected by placing cups filled with larval rearing water and lined with filter paper (diameter $90 \mathrm{~mm}$ ) into cages of mosquitoes that were blood fed 4-5 days earlier. Filter papers were replaced every $0.5-1 \mathrm{~h}$. Eggs ( $<1.5 \mathrm{hr}$ old, light gray in color) were lined up on filter paper and transferred to a cover slip with double-sided tape. Eggs were left to desiccate for 1-2 min before being covered in halocarbon oil 700 (Sigma Aldrich, Castle Hill, NSW Australia). Eggs were injected using a MINJ-1000 microinjection system (Tritech Research, Los Angeles, CA, USA) and left in oil for $2 \mathrm{hr}$. Eggs were gently removed from the oil using a fine paintbrush, rinsed in water and placed on a moist piece of filter paper. Eggs were conditioned at $~ 80 \%$ humidity then hatched $3 \mathrm{~d}$ post-injection by submerging filter papers in containers filled with RO water, a few grains of yeast and one tablet of fish food. Hatching larvae were reared to adulthood and $F_{0}$ females were crossed to males from an 
uninfected population (QL), blood fed, isolated for oviposition, and screened for wAlbB infection after producing viable offspring. This process was repeated for three further generations, with only progeny from Wolbachia-positive females contributing to the next generation. The population was closed (with no further backcrossing) once the wAlbB infection reached fixation. We used LAMP assays for rapid detection of WAlbB during microinjection experiments according to JASPER et al. (2019).

\section{Antibiotic curing}

To generate uninfected Ae. aegypti mosquitoes with matching mitochondrial haplotypes and nuclear backgrounds, we cured the wAlbB infection using antibiotic treatment, with untreated populations reared in parallel. wAlbB Au (generation 5 post-microinjection) adults were fed $2 \mathrm{mg} / \mathrm{mL}$ tetracycline hydrochloride ( $\geq 95 \%$, Sigma-Aldrich P/L, Castle Hill NSW, Australia) in a 10\% sucrose solution for 10 days before blood feeding. Larvae from the next generation were reared in a solution of $50 \mu \mathrm{g} / \mathrm{L}$ tetracycline hydrochloride according to ENDERSBY-HARSHMAN et al. (2019). This process was repeated for a total of three generations of adult treatment and two generations of larval treatment. After the third generation of adult treatment, 150 females from the treated population were blood fed and isolated for oviposition, with larvae from Wolbachia-negative mothers pooled to generate uninfected populations. To control for potential effects of drift or inbreeding (Ross et al. 2019a), wAlbB-infected (untreated) and uninfected (tetracycline-cured) females from each population were crossed to wild-type uninfected males of Australian background for one generation. The resulting populations were divided into two replicate populations for backcrossing (Figure 1A).

\section{Backcrossing}

To generate populations with different combinations of nuclear background (Australian or Malaysian) and Wolbachia infection status, we performed backcrosses with an uninfected Malaysian population (Figure 1B). wAlbB Au and Uninfected Au females were crossed to uninfected Malaysian (MY) males. Two replicate populations for each combination were backcrossed independently for a total of four populations. Each generation, 200 female pupae and 200 male pupae were sexed and left to emerge in separate cages. Sexes were confirmed following adult emergence and females were aspirated into cages of males to mate. This process was repeated for two additional generations, resulting in an estimated 87.5\% similarity to the target background. Four additional populations (two replicates each of wAlbB Au and Uninfected $\mathrm{Au}$ ) were not backcrossed. Instead, these were maintained at a census size of 400 individuals each generation. Before experiments commenced, all populations were screened for wAlbB infection as well as their COI haplotype (see below) to check for contamination during antibiotic curing and backcrossing. 
bioRxiv preprint doi: https://doi.org/10.1101/2021.06.25.450002; this version posted June 26, 2021. The copyright holder for this preprint (which was not certified by peer review) is the author/funder, who has granted bioRxiv a license to display the preprint in perpetuity. It is made available under aCC-BY-NC 4.0 International license.

A

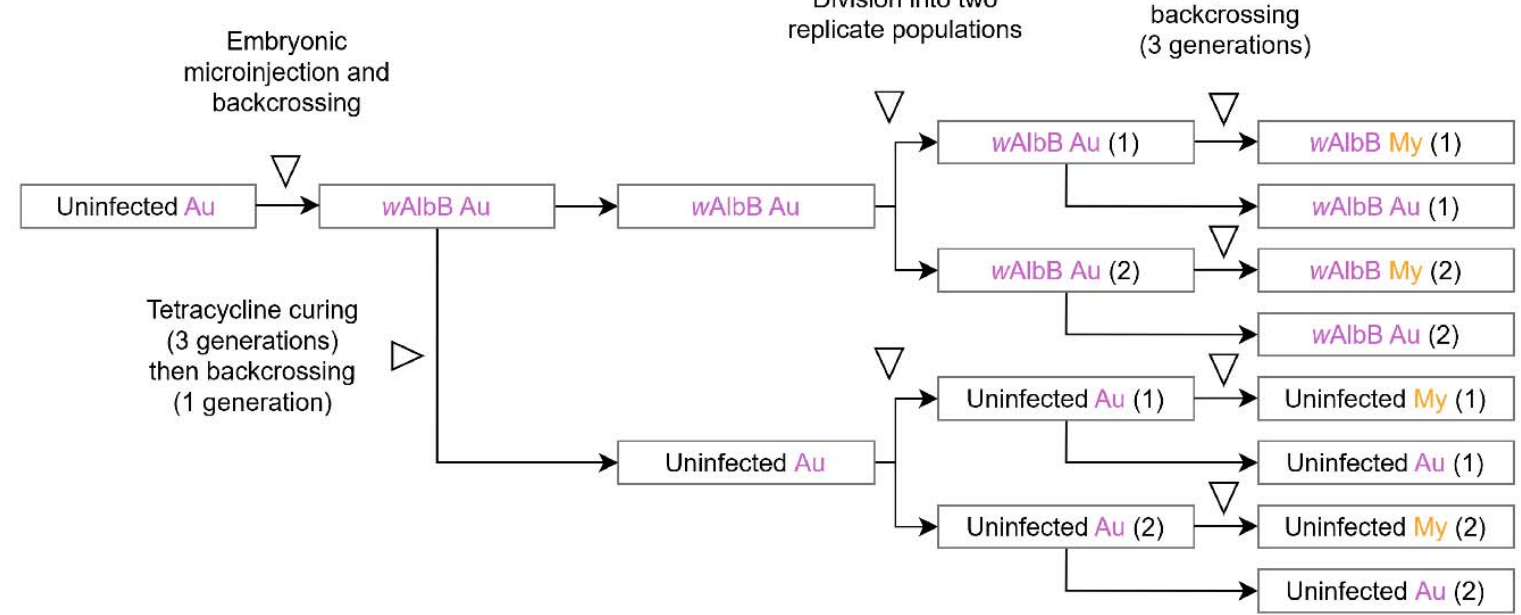

B
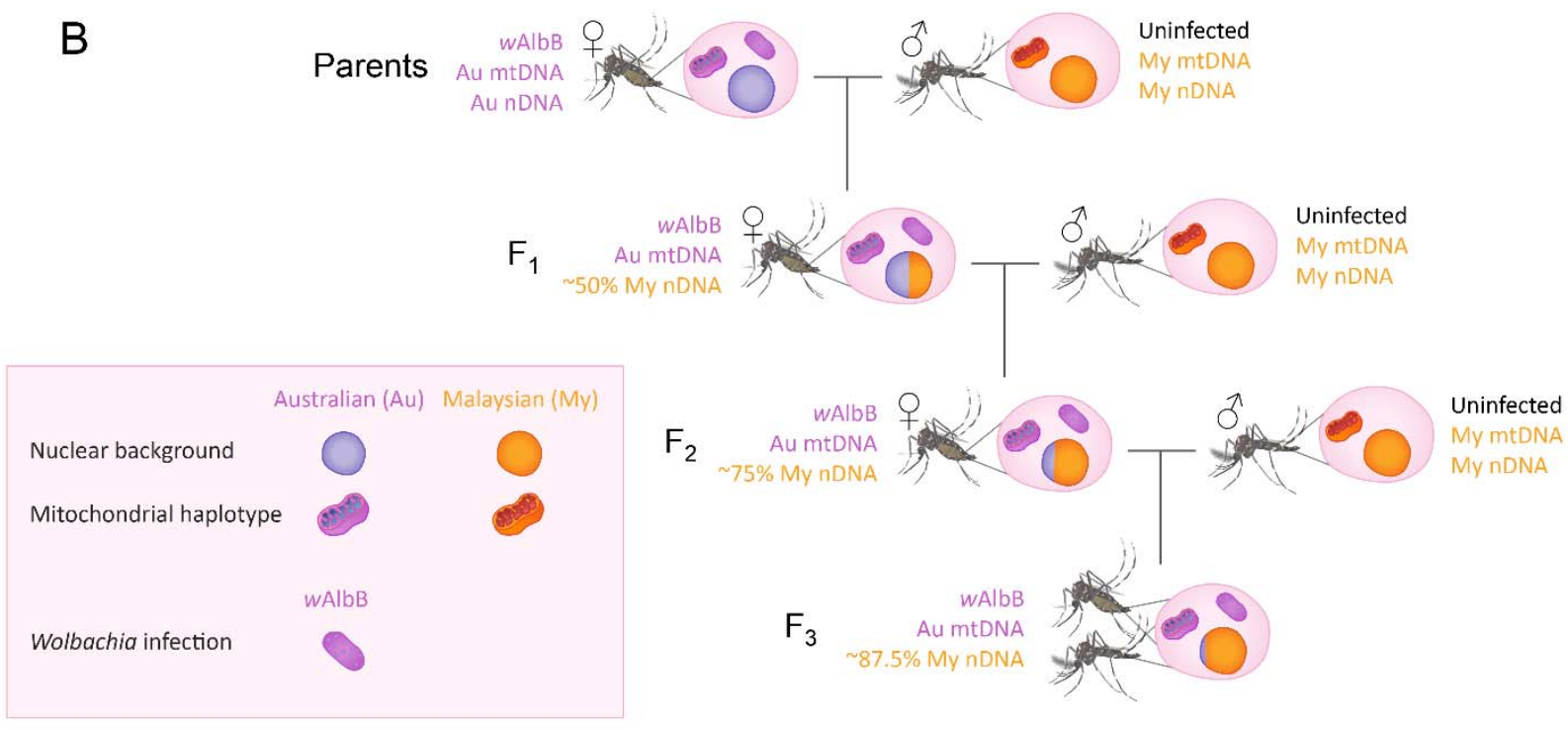

Figure 1. Experimental design and backcrossing scheme. (A) Experimental design showing the division of populations before and after tetracycline curing and before backcrossing. (B) Backcrossing scheme showing the introgression of the wAlbB infection and Australian (Au) mitochondria into a Malaysian (My) nuclear background. wAlbB Au females were crossed to uninfected Malaysian (MY) males for three consecutive generations. Backcrossing was also conducted with Uninfected Au females into a Malaysian background. Mosquito illustrations by Ana Ramírez (2018). 


\title{
DNA level characterization of Wolbachia
}

\author{
Whole genome sequencing
}

We sequenced the whole Wolbachia genome of the $\mathrm{WAlbB}^{\complement}$ transinfection generated in this study after 11 generations post-transinfection. Genomic DNA was extracted from a pooled sample of 5 individuals using a DNeasy Blood \& Tissue kit (Qiagen, Hilden, Germany). Extracted DNA was randomly fragmented to a size of $350 \mathrm{bp}$ then end-polished, A-tailed, and ligated with Illumina sequencing adapters using a NEBNext ${ }^{\circledR}$ Ultra ${ }^{\text {TM }}$ DNA Library Prep Kit (New England Biolabs, Ipswich MA, USA), and further PCR enriched with primers of $P 5$ and $P 7$ oligos. The PCR products which comprised the final libraries were purified (AMPure XP system, Beckman Coulter Life Sciences, Indianapolis IN, USA) and subjected to quality control tests that included size distribution by Agilent 2100 Bioanalyzer (Agilent Technologies, CA, USA), and molarity measurement using real-time PCR. The libraries were then pooled and sequenced on a NovaSeq 6000 (Illumina) using $2 \times 150$ bp chemistry by Novogene HK Company Limited, Hong Kong.

\section{Reference genome assembly}

Quality filtering of sequencing reads was performed with Trimmomatic (BOLGER et al. 2014), with the following parameter settings: leading $=3$; trailing $=3$; slidingwindow $=4: 15$; inlen $=71$. Reads were aligned to a wAlbB reference genome (SINHA et al. (2019), GenBank accession CP031221.1) using the Burrows-Wheeler Aligner (LI 2013), with the bwa mem algorithm and default parameter settings. Subsequent quality filtering and variant calling was performed with SAMtools and BCFtools (LI et al. 2009; DANECEK et al. 2021). PCR duplicates were excluded from downstream analyses by soft masking. Reads with a MAPQ score $<25$ were removed from the alignment, except for reads with MAPQ $=0$, which were permitted to allow for mapping to repetitive regions. Genomic likelihoods were calculated using a maximum of 2000 reads per position. For variant calling, ploidy was set to haploid. The variant call output was used to create a consensus nucleotide sequence, wherein genome positions with coverage $<5$ were masked as ' $\mathrm{N}$ '. The consensus sequence was compared to the reference genome and two additional wAlbB genomes, originating from Hainan, China and Florida, USA (KULKARNI et al. 2019b; KULKARNI et al. 2019a). Polymorphic nucleotide positions were identified by aligning the genome sequences with Geneious v 9.1.8 (https://www.geneious.com). Regions of the Hainan and Florida wAlbB genomes displaying anomalous patterns of nucleotide variation, suggestive of localized sequence contamination, were annotated by eye.

\section{Phylogenomic analysis}

Genome assemblies and corresponding protein annotation data were obtained from GenBank (Appendix S1). Thirteen representative supergroup B genomes from various arthropod hosts were included in the analysis, including three publicly available wAlbB genomes (KULKARNI et al. 2019a; KULKARNI et al. 2019b; SINHA et al. 2019) and two supergroup A outgroup genomes (Appendix S1). 
Orthofinder v2.5.2 (EMMS AND KELLY 2019) was used to identify a core set of orthologs that were present as single-copy genes in all of the Wolbachia genomes included in the analysis (Appendix S2). Ortholog nucleotide sequences were aligned with MAFFT v7.475 (KATOH AND STANDLEY 2013) using the 'auto' setting. Alignments were trimmed with Gblocks v0.91b (CASTRESANA 2000) using the following settings: minimum number of sequences for a conserved position $=$ seqs $/ 2+1$, minimum number of sequences for a flank = seqs $/ 2+1$, maximum number of contiguous non-conserved positions $=8$, minimum block length $=5$, gap allowed $=$ none. Sixteen ortholog alignments were excluded from subsequent analyses due to possible sequence contamination in one sample (CP041923.1; see above). For phylogenetic analysis, the individual orthogroup alignments were concatenated to form a single alignment comprised of 459 orthogroups and 382,520 nucleotide positions. Maximum likelihood trees were constructed with RAXML-HPC v8.2.12 (STAMATAKIS 2014) on XSEDE, using a GTR-GAMMA model with rapid bootstrapping (100 inferences). Bootstrap scores were plotted onto the best scoring ML tree. RAXML-HPC was accessed through the CIPRES Science Gateway (MILLER et al. 2010).

\section{COI sequencing}

To confirm the successful transfer of the wAlbB infection to Ae. aegypti with an Australian mitochondrial haplotype, we performed $\mathrm{CO}$ sequencing on six individuals from the original wAlbB population described in XI et al. (2005b) as well as nine individuals from the wAlbB Au population generated through microinjection in the current study. To check for contamination following backcrossing, we performed $\mathrm{COI}$ sequencing from three individuals from each of the 8 populations used in experiments (Table 1). We performed COI amplicon sequencing following YEAP et al. (2016). Samples were analysed for a 750bp region within the COI region (positions 1994-2743 on GenBank: EU352212.1) using forward primer UEA5 5'AGTTTTAGCAGGAGCAATTACTAT3' and reverse primer UEA10 5'TCCAATGCACTAATCTGCCATATTA3' (LUNT et al. 1996). PCR amplicons from individuals were sequenced in both forward and reverse directions using Sanger Sequencing (Macrogen, Inc., Geumcheongu, Seoul, South Korea). The sequenced $750 \mathrm{bp}$ region was analysed using Geneious 9.1.8 to investigate SNP variation among samples.

\section{Wolbachia detection and density}

We used real-time PCR assays (LEE et al. 2012; AXFORD et al. 2016) to confirm the presence or absence of Wolbachia infection and estimate relative density using the Roche LightCycler ${ }^{\circledR} 480$. Genomic DNA was extracted using $250 \mu \mathrm{L}$ of $5 \%$ Chelex 100 Resin (Bio-Rad laboratories, Hercules CA) and $3 \mu \mathrm{L}$ of Proteinase K (20 mg/mL) (Roche Diagnostics Australia Pty. Ltd., Castle Hill New South Wales, Australia). Tubes were incubated for $60 \mathrm{~min}$ at $65^{\circ} \mathrm{C}$ then $10 \mathrm{~min}$ at $90^{\circ} \mathrm{C}$. Three primer sets were used to amplify markers specific to mosquitoes, Ae. aegypti and wAlbB. For mosquitoes carrying the wMel infection, Wolbachia density was determined using w1 primers (LEE et al. 2012). Relative Wolbachia densities were determined by subtracting the crossing point $(\mathrm{Cp})$ value of the Wolbachia-specific marker from the $\mathrm{Cp}$ 
value of the mosquito-specific marker. Differences in Cp were averaged across 2-3 consistent replicate runs, then transformed by $2^{n}$. For the maternal transmission experiment we required only presenceabsence data, so a single run was performed per sample (with any negative samples repeated to confirm a lack of infection).

\section{Experimental comparisons}

\section{Life history parameters}

To evaluate the effects of Wolbachia infection status and nuclear background on mosquito life history, we performed phenotypic assessments of the 8 populations generated through backcrossing (Table 1). Eggs ( $<1$ week old) from each population were hatched in trays filled with $3 \mathrm{~L}$ of RO water, a few grains of yeast, and one tablet of fish food. One day after hatching, 100 larvae were counted into trays filled with $500 \mathrm{~mL}$ of RO water, with 5 replicate trays per population. Larvae were provided with fish food $a d$ libitum until pupation. To determine the average larval development time and survival to pupation for each tray, pupae were counted and sexed twice per day (in the morning and evening). Pupae were removed from larval development trays, pooled across replicate trays, and placed in an open container of water inside a BugDorm- 4F2222 (13.8 L) cage for adults to emerge.

Groups of adults were stored within $24 \mathrm{hr}$ of emergence for Wolbachia density and wing length measurements. One wing each from 20 males and 20 females per population was dissected and measured for length according to Ross et al. (2016) as an estimate of body size, with damaged wings excluded from analysis. Adults from the four wAlbB-infected populations ( 20 females and 20 males per population) were screened with real-time PCR for Wolbachia density estimation (as described above). The remaining adults were provided with a $10 \%$ sucrose solution which was then removed $24 \mathrm{hr}$ prior to blood feeding. Females (6-7 d old) were fed human blood via Hemotek ${ }^{\circledR}$ membrane feeders. Twenty engorged females per population were isolated for oviposition in $70 \mathrm{~mL}$ specimen cups with mesh lids that were filled with $15 \mathrm{~mL}$ of larval rearing water and lined with a strip of sandpaper (Norton Master Painters P80 sandpaper, Saint-Gobain Abrasives Pty. Ltd., Thomastown, Victoria, Australia). Eggs were collected four days after blood feeding, partially dried, and then hatched three days after collection by submerging sandpaper strips in containers filled with RO water, a few grains of yeast and one tablet of fish food. Female fecundity was determined by counting the total number of eggs on each sandpaper strip, while hatch proportions were determined by dividing the number of hatched eggs (with a clearly detached egg cap) by the total number of eggs per female. Females that did not lay eggs or died before laying eggs were excluded from the analysis.

\section{Quiescent egg viability}

Blood-fed females remaining in cages from the previous experiment were used to test quiescent egg viability. Six cups filled with larval rearing water and lined with sandpaper strips were placed inside each 
cage. Eggs were collected five days after blood feeding, partially dried, then placed in a sealed chamber with an open container of saturated potassium chloride solution to maintain a constant humidity of 84\%. When eggs were 1, 4, 7, 10, 13 and 16 weeks old, small sections of each sandpaper strip were removed and submerged in water with a few grains of yeast to hatch. Four to six replicate batches of eggs were hatched per population at each time point, with 40-125 eggs per batch. Hatch proportions were determined by dividing the number of hatched eggs (with a clearly detached egg cap) by the total number of eggs per female.

\section{Cytoplasmic incompatibility and Wolbachia density following heat stress}

We measured Wolbachia density and cytoplasmic incompatibility in adults after being exposed to cyclical heat stress during the egg stage. Eggs were collected from Wolbachia-infected populations (one replicate population each from wAlbB Au, wAlbB My and wMel). Four days after collection, batches of 40-60 eggs were tipped into $0.2 \mathrm{~mL}$ PCR tubes (12 replicate tubes per population) and exposed to cyclical temperatures of $29-39^{\circ} \mathrm{C}$ for $7 \mathrm{~d}$ in Biometra TProfessional TRIO 48 thermocyclers (Biometra, Göttingen, Germany) according to KONG et al. (2016) and Ross et al. (2019b). Eggs of the same age from each population, as well as Uninfected Au eggs, were kept at $26^{\circ} \mathrm{C}$. Eggs held at $29-39^{\circ} \mathrm{C}$ and $26^{\circ} \mathrm{C}$ were hatched synchronously and larvae were reared at a controlled density (100 larvae per tray of $500 \mathrm{~mL}$ water). Pupae were sexed and 15 males and 15 females per population and temperature treatment were stored in absolute ethanol within $24 \mathrm{hr}$ of emergence for Wolbachia density estimation (see Wolbachia detection and density). The remaining pupae were sexed and released into BugDorm-4S1515 $(5.4 \mathrm{~L}$ ) cages (with each sex, temperature treatment and population held in separate cages) for cytoplasmic incompatibility crosses.

We established two sets of crosses to 1 . test the ability of Wolbachia-infected males to induce cytoplasmic incompatibility and 2. test the ability of Wolbachia-infected females to restore compatibility with Wolbachia-infected males. In the first set, Uninfected Au females (untreated) were crossed with Wolbachia-infected males from each temperature treatment by aspirating females into cages of males. In the second set, Wolbachia-infected females from each temperature treatment were crossed with wAlbB Au males, except for wMel females which were crossed to wMel (untreated) males. Females (5-7 $\mathrm{d}$ old, starved for $24 \mathrm{hr}$ ) were blood-fed and 20 females per cross were isolated for oviposition. Hatch proportions were determined by dividing the number of hatched eggs (with a clearly detached egg cap) by the total number of eggs per female. Females that did not lay eggs or died before laying eggs were excluded from the analysis.

\section{Wolbachia maternal transmission}

We tested the fidelity of Wolbachia maternal transmission in each nuclear background. Females from the wAlbB Au and wAlbB My populations (one replicate population each) were crossed to Uninfected $\mathrm{Au}$ males. Females (5-7 d old, starved for $24 \mathrm{hr}$ ) were blood-fed, isolated for oviposition, then stored in 
absolute ethanol after laying eggs. Offspring from each female were hatched, reared in trays of $500 \mathrm{~mL}$ RO water, then stored $4 \mathrm{~d}$ after hatching. We tested ten mothers and ten offspring per mother for the presence of wAlbB using real-time PCR (see Wolbachia detection and density).

\section{Statistical analysis}

All data were analyzed using SPSS Statistics version 24.0 for Windows (SPSS Inc, Chicago, IL). Data sets were tested for normality with Shapiro-Wilk tests and transformed where appropriate (egg hatch proportion and survival to pupa data were logit transformed). Life history, quiescent egg viability and Wolbachia density data were analyzed with general linear (mixed effect) models (GLMs). We tested the effects of Wolbachia infection (wAlbB-infected or uninfected), nuclear background (Au or My) and interactions between Wolbachia infection and nuclear background. Replicate populations were pooled for analysis when effects of replicate population exceeded a P-value of 0.1 in prior analyses. Where interaction terms were not-significant, the terms were dropped and the models were rerun without interactions. Data for each sex were analyzed separately. For quiescent egg viability, hatch proportions differed substantially between wAlbB-infected and uninfected populations. We therefore ran separate GLMs for wAlbB-infected and uninfected populations, with egg storage duration included as a continuous covariate for this trait. Replicate population (nested within nuclear background) was included as a random factor but was not significant in any instance. Quadratic egg storage duration was also included as a factor in the GLM in case of a non-linear relationship between egg hatch proportion and storage duration in these populations. We used chi-squared tests to determine whether sex-ratios for each population differed significantly from 1:1. Bonferroni corrections were performed on $\mathrm{P}$ values when multiple traits were measured in the same experiment.

For Wolbachia density, untransformed data (i.e. differences in $\mathrm{Cp}$ between Wolbachia and mosquito markers, before $2^{n}$ transformation) were used for analyses to test for effects of nuclear background and the temperature treatment $\left(26\right.$ or $26-39^{\circ} \mathrm{C}$ ) as a factor. We were unable to perform direct comparisons between wMel and wAlbB strains due to using different markers for each strain; we therefore excluded wMel from the overall analysis but presented it graphically. Egg hatch proportions from cytoplasmic incompatibility crosses were analysed with Mann-Whitney U tests.

\section{Results}

\section{Genomic analysis of the $w_{A l b B^{\alpha}}$ transinfection}

We sequenced the whole Wolbachia genome of the $\mathrm{WAlbB}^{\complement}$ transinfection generated in this study. Its genome is almost identical to the wAlbB reference genome (SINHA et al. 2019), differing by only four single nucleotide variants (SNVS), suggesting that few genetic changes have occurred since wAlbB was first transferred to Ae. aegypti over 15 years ago (XI et al. 2005b). The genomes of the wAlbB variants 
from Hainan, China and Florida, USA were more divergent, each differing from the wAlbB reference genome by more than 100 SNPs, substitutions and small indels, and by the presence of at least one large deletion (>100 bp) for the Hainan variant and at least four large deletions for the Florida variant (Appendix S3).

We performed a phylogenomic analysis with a core set of protein-encoding gene ortholog sequences conserved as single-copy genes across various Wolbachia supergroup B infections, including four wAlbB variants and two supergroup A outgroup strains. The wAlbB variants formed a monophyletic group that shared a most recent common ancestor with the Wolbachia strains WNo, wMau and WTpre from Drosophila simulans, Drosophila mauritiana and Trichogramma pretiosum respectively (Figure 2A). A separate analysis of only the wAlbB sequences placed the AAlbB $^{\complement}$ transinfection in a cluster with the wAlbB reference variant, in agreement with the above sequencing results (Figure 2B). Relative to the $w A l b B$ reference and $w_{A l b B}{ }^{Q}$ transfection genomes, the Hainan variant was slightly less divergent than the Florida variant, consistent with the pattern of nucleotide variation observed for the whole genome sequences (Appendix S3). 


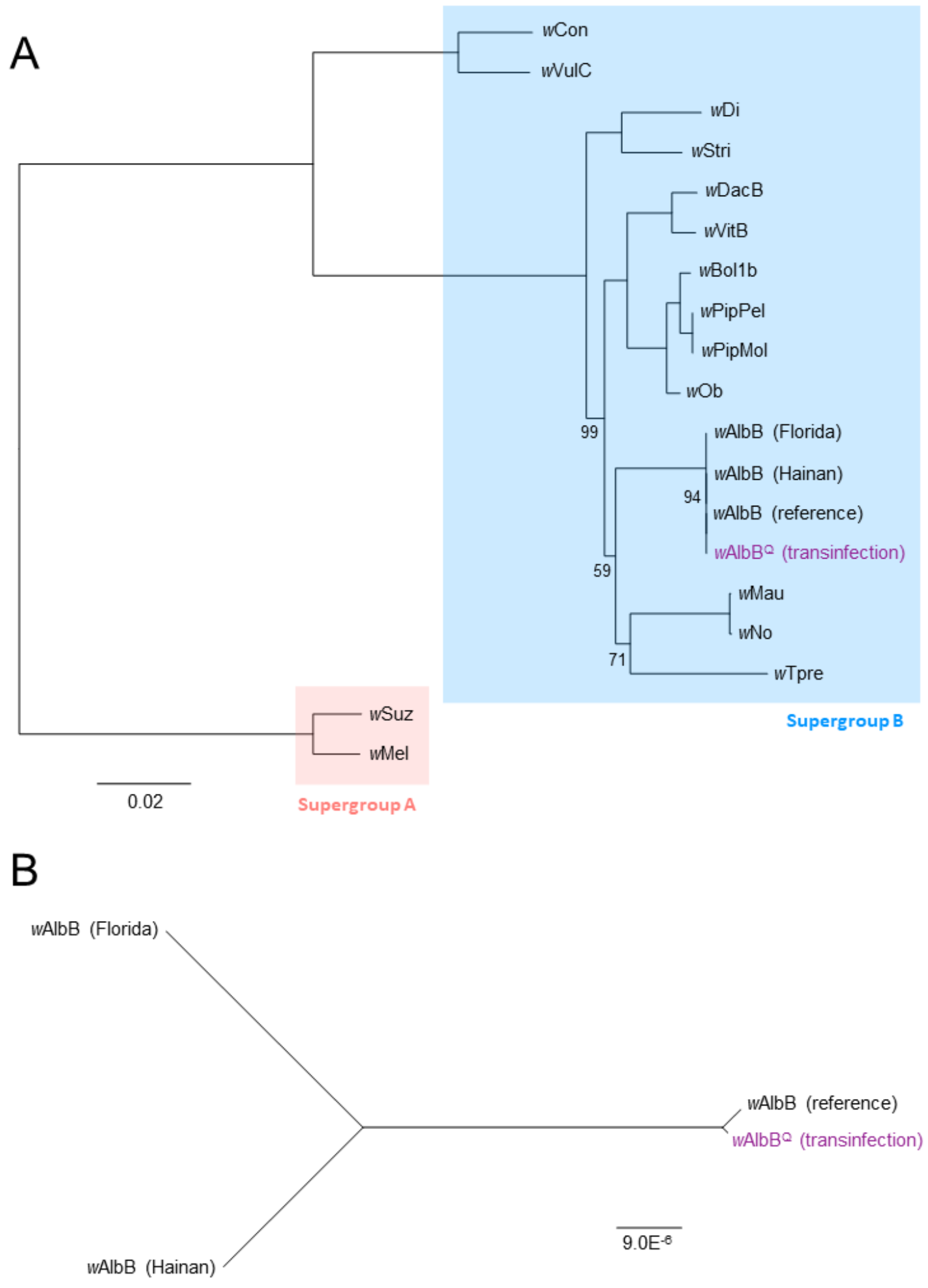

Figure 2. Phylogenomic analyses of wAlbB variants. Maximum likelihood trees were constructed with RAXML-HPC using a concatenated alignment of 459 gene orthologs, comprised of 382,520 nucleotide positions. Bootstrap values are 100 unless shown otherwise; scale bars = number of substitutions per 
site. The AAlbB $^{Q}$ transinfection is highlighted in purple. For the analysis of $(\mathrm{A})$ Wolbachia from different host species, the alignment comprised 9,469 distinct patterns and a rooted tree was built using two supergroup A sequences as outgroups; for the analysis of (B) wAlbB variants, an unrooted tree was built using an alignment with 23 distinct patterns.

\section{Life history traits are influenced by wAlbB infection and nuclear background}

To test the contributions of Wolbachia infection and nuclear background to mosquito life history, we generated 8 divergent populations using a combination of embryonic microinjection, antibiotic curing and backcrossing (Table 1, Figure 1). wAlbB was successfully introduced to mosquitoes with an Australian mitochondrial haplotype through cytoplasm transfer, with the resulting transinfection $\left(\right.$ A AlbB $\left.^{Q}\right)$ showing stable transmission by the third generation (Table S1). Following backcrossing, all 8 populations had $\mathrm{COI}$ sequences matching their expected mitochondrial haplotype (Table 1, Table S2).

Cohorts of mosquitoes from each population were evaluated for life history traits including larval development time, survival to pupa, fecundity, egg hatch proportion and body size (Figure 3).

Development time and survival to pupa were not significantly affected by Wolbachia infection or nuclear background (Table 2). Nuclear background had a substantial effect on fecundity (Table 2), with Malaysian background mosquitoes laying more eggs than those with an Australian background (Figure $3 E)$. The wAlbB infection reduced the proportion of eggs hatching, but there was no significant effect of nuclear background (Table 2, Figure 3F). Mosquitoes with a Malaysian background tended to have larger wings than mosquitoes with an Australian background (Figure 3G, H), but this effect was only significant in males (Table 2). Sex ratios of pupae (Figure 3D) did not deviate significantly from 1:1 (Chi-square: $\mathrm{df}=$ 9 , all $P>0.05$ ) for any population. Overall, our results indicate clear effects of nuclear background on fecundity and wing length, with wAlbB infection inducing a cost to egg hatch in both nuclear backgrounds.

Table 2. GLMs for life history parameters of wAlbB-infected and uninfected Aedes aegypti of Australian ( $\mathrm{Au}$ ) and Malaysian (My) backgrounds. $\mathrm{P}$ values in bold are significant following Bonferroni correction (Adjusted $\alpha$ : 0.00556).

\begin{tabular}{|l|l|l|l|l|l|l|}
\hline Trait & Factor & $\begin{array}{l}\text { Type III Sum } \\
\text { of Squares }\end{array}$ & $\begin{array}{l}\text { Degrees } \\
\text { of } \\
\text { freedom }\end{array}$ & $\begin{array}{l}\text { Mean } \\
\text { Square }\end{array}$ & F & P \\
\hline $\begin{array}{l}\text { Female development } \\
\text { time }\end{array}$ & Wolbachia infection & 0.016 & 1 & 0.016 & 1.279 & 0.265 \\
\hline & Nuclear background & 0.085 & 1 & 0.085 & 6.729 & 0.014 \\
\hline & Error & 0.468 & 37 & 0.013 & & \\
\hline Male development & Wolbachia infection & 0.032 & 1 & 0.032 & 1.175 & 0.285 \\
\hline
\end{tabular}


bioRxiv preprint doi: https://doi.org/10.1101/2021.06.25.450002; this version posted June 26, 2021. The copyright holder for this preprint (which was not certified by peer review) is the author/funder, who has granted bioRxiv a license to display the preprint in perpetuity. It is made available under aCC-BY-NC 4.0 International license.

\begin{tabular}{|c|c|c|c|c|c|c|}
\hline \multicolumn{7}{|l|}{ time } \\
\hline & Nuclear background & 0.123 & 1 & 0.123 & 4.571 & 0.039 \\
\hline & Error & 0.995 & 37 & 0.027 & & \\
\hline \multirow[t]{3}{*}{ Survival to pupa } & Wolbachia infection & 0.003 & 1 & 0.003 & 1.379 & 0.248 \\
\hline & Nuclear background & 0.000 & 1 & 0.000 & 0.177 & 0.677 \\
\hline & Error & 0.075 & 37 & 0.002 & & \\
\hline \multirow[t]{3}{*}{ Fecundity } & Wolbachia infection & 5.663 & 1 & 5.663 & 0.020 & 0.889 \\
\hline & Nuclear background & 4573.419 & 1 & 4573.419 & 15.874 & $<0.0001$ \\
\hline & Error & 42351.708 & 147 & 288.107 & & \\
\hline \multirow[t]{3}{*}{ Egg hatch proportion } & Wolbachia infection & 0.595 & 1 & 0.595 & 10.911 & 0.001 \\
\hline & Nuclear background & 0.060 & 1 & 0.060 & 1.096 & 0.297 \\
\hline & Error & 7.856 & 144 & 0.055 & & \\
\hline \multirow[t]{3}{*}{ Female wing length } & Wolbachia infection & 0.007 & 1 & 0.007 & 0.920 & 0.339 \\
\hline & Nuclear background & 0.034 & 1 & 0.034 & 4.538 & 0.035 \\
\hline & Error & 1.163 & 157 & 0.007 & & \\
\hline \multirow[t]{3}{*}{ Male wing length } & Wolbachia infection & 0.001 & 1 & 0.001 & 0.377 & 0.540 \\
\hline & Nuclear background & 0.088 & 1 & 0.088 & 27.554 & $<0.0001$ \\
\hline & Error & 0.544 & 171 & 0.003 & & \\
\hline
\end{tabular}


A
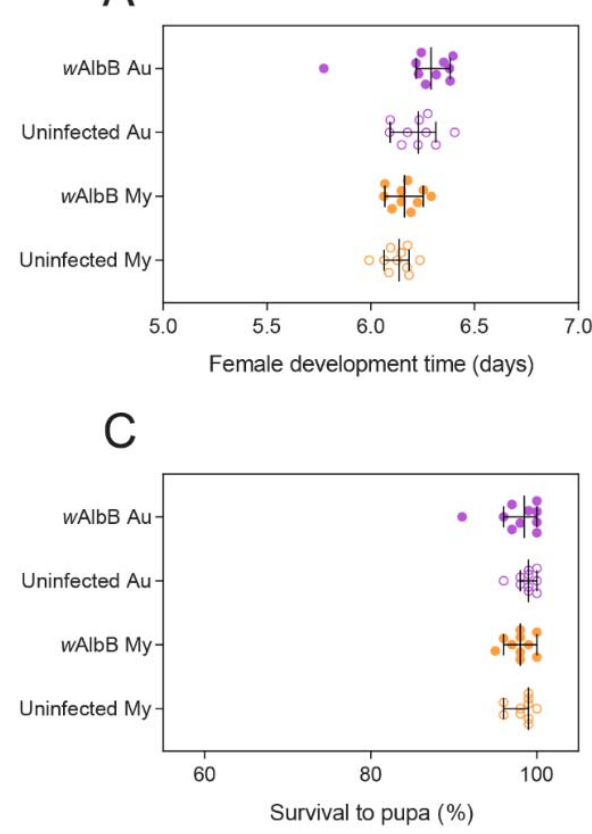

E

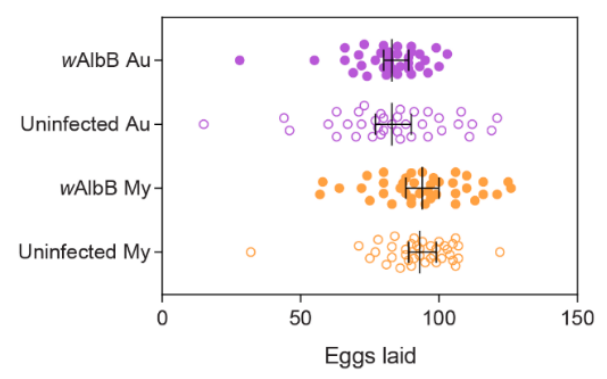

G

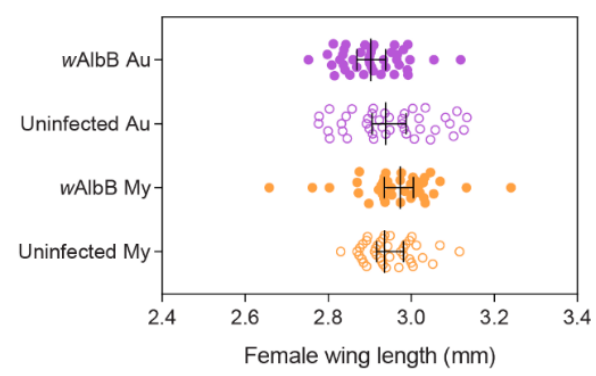

B

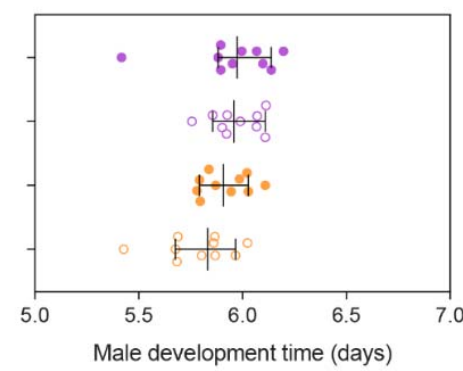

D

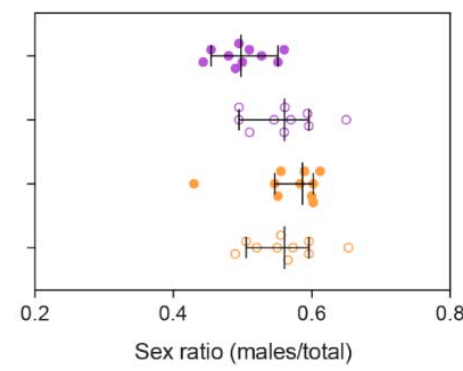

$\mathrm{F}$

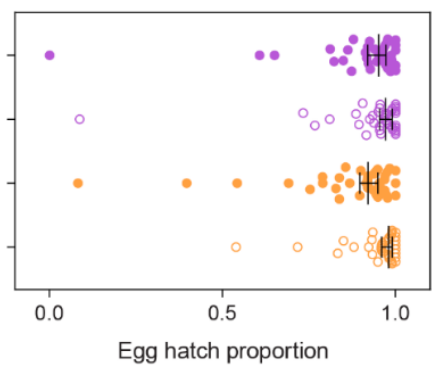

$\mathrm{H}$

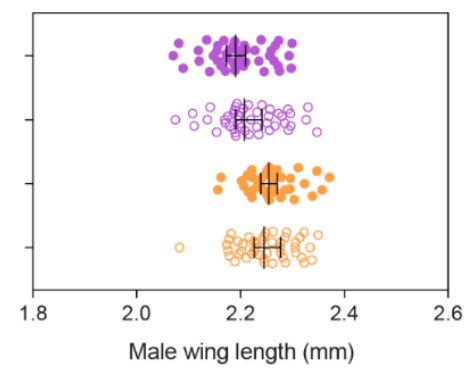

Figure 3. Life history parameters of wAlbB-infected and uninfected Aedes aegypti of Australian (Au) and Malaysian (My) backgrounds. Populations were evaluated for the following traits: larval development time for (A) females and (B) males, (C) percent survival to the pupal stage, (D) sex ratio, (E) female fecundity, $(F)$ egg hatch proportion and wing length of $(G)$ female and $(H)$ males. Data from two replicate populations were pooled for visualization. See table 1 for a description of each population. Each point represents data averaged across a replicate container of 100 individuals (A-D) or data from individual mosquitoes (panels E-H). Medians and 95\% confidence intervals are shown in black lines. 


\section{Quiescent egg viability depends on mosquito nuclear background and wAlbB infection}

Stored eggs from each population were hatched every three weeks to determine quiescent egg viability. wAlbB infection greatly reduced quiescent egg viability in both Australian and Malaysian backgrounds (Figure 4). By week 16, hatch proportions for wAlbB-infected populations approached zero while hatch proportions for uninfected populations exceeded $40 \%$. In uninfected populations, a $L M$ indicated a significant interaction between background and week $\left(\mathrm{F}_{(1,118)}=13.193, \mathrm{P}<0.001\right)$ due to the sharp decrease in viability in the Malaysian background. There was also an effect of week in this analysis $\left(F_{i 1}\right.$, ${ }_{118)}=98.967, \mathrm{P}<0.001$ ). Eggs with an Australian background had higher hatch proportions (median 0.819) than eggs with a Malaysian background (median 0.445 ) by the end of the experiment. In wAlbBinfected populations where we did not consider the data from week 16 , there was also a significant interaction between background and week $\left(F_{(1,93)}=22.948, P<0.001\right)$ due to the sharper decrease in viability in the Malaysian background along with an effect of week $\left(F_{(1,93)}=368.012, P<0.001\right)$.

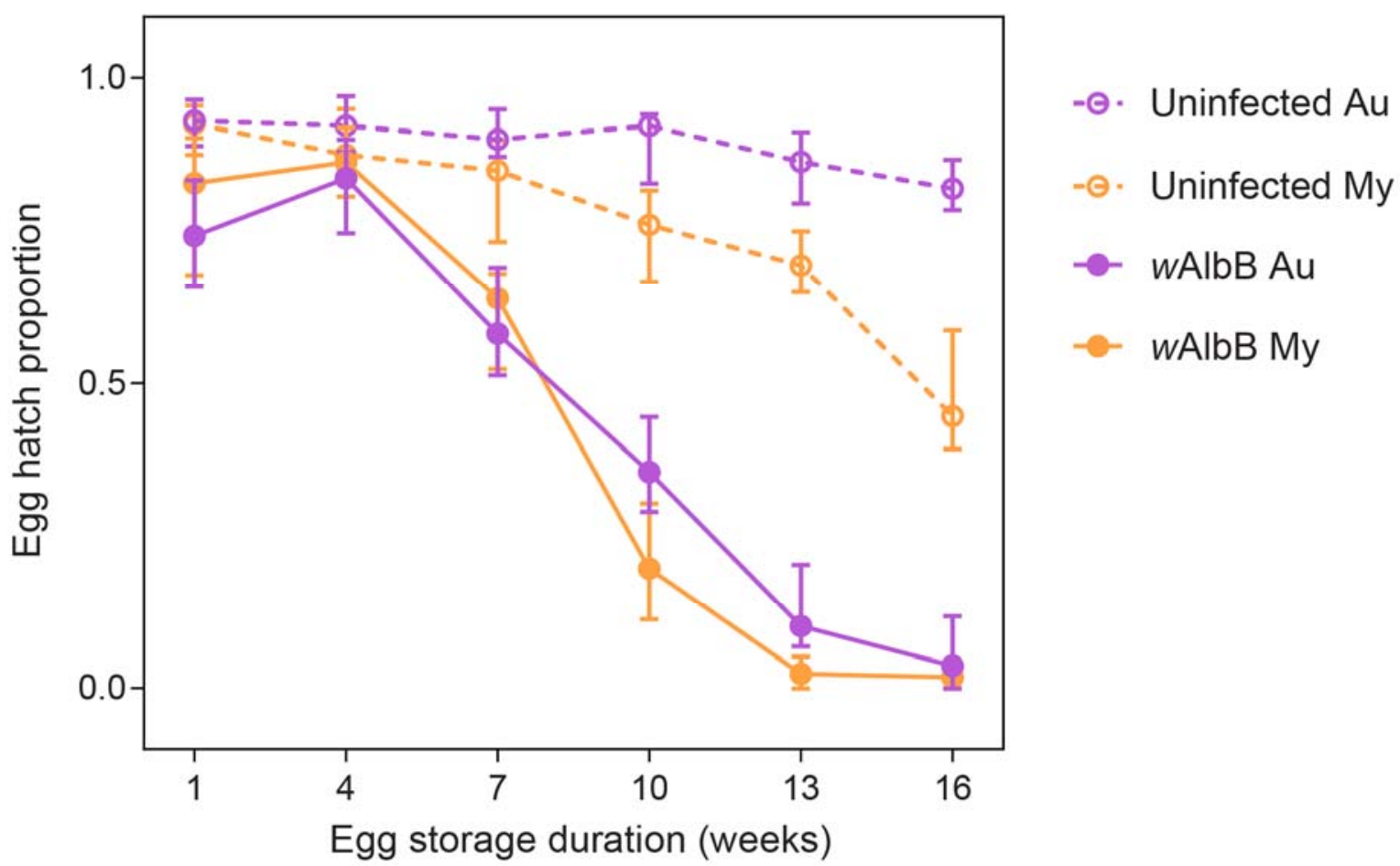

Figure 4. Quiescent egg viability of wAlbB-infected and uninfected Aedes aegypti of Australian (Au) and Malaysian (My) backgrounds. Data from two replicate populations were pooled for visualization. Symbols show median egg hatch proportions while error bars show 95\% confidence intervals. 


\section{Nuclear background influences wAlbB density}

We estimated Wolbachia density in whole adults from the wAlbB Au and wAlbB My populations (Figure 5). Wolbachia density was higher in Australian mosquitoes than Malaysian mosquitoes for both females (GLM: $\left.F_{1,82}=6.752, P=0.011\right)$ and males $\left(F_{1,72}=7.956, P=0.006\right)$.
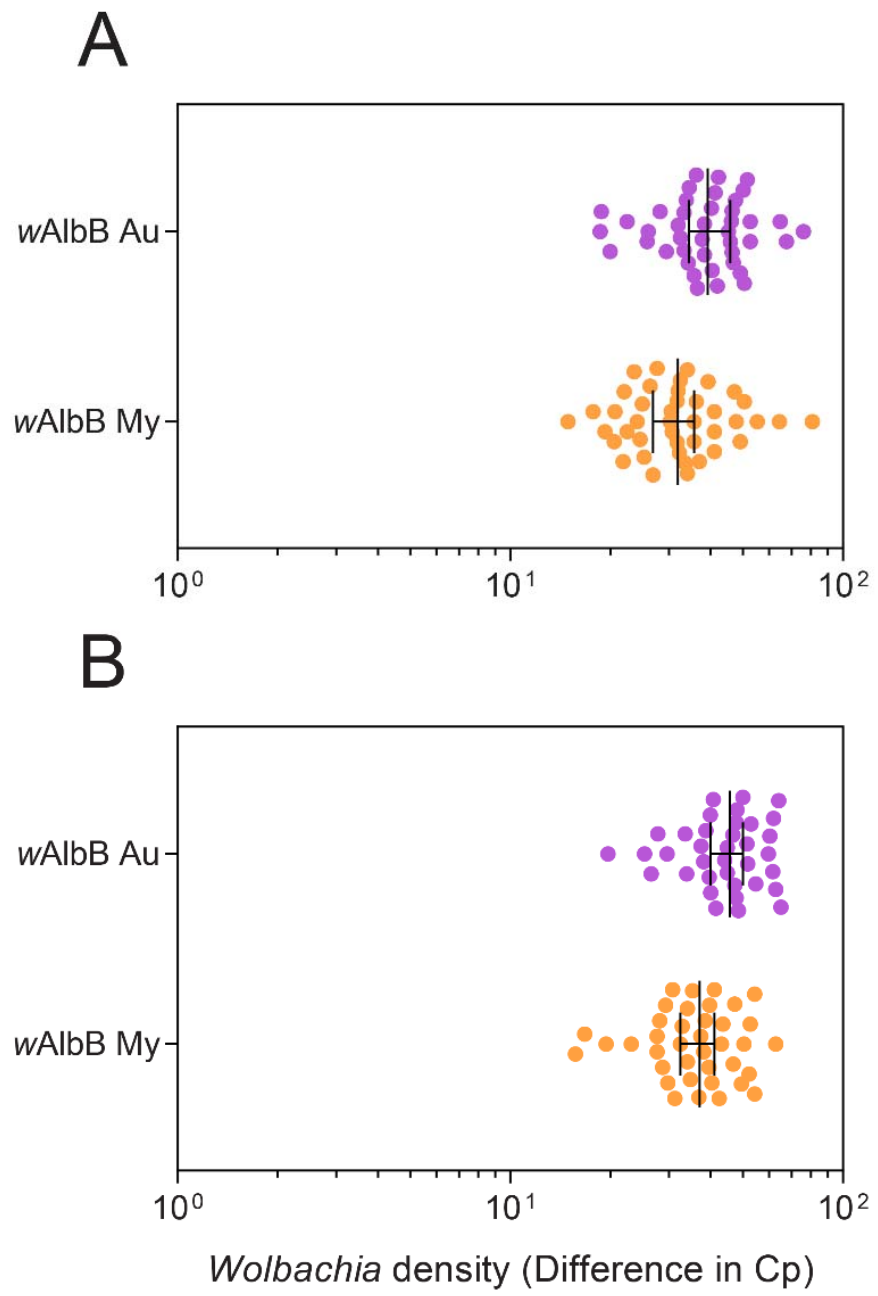

Figure 5. Female (A) and male (B) Wolbachia density in wAlbB-infected Aedes aegypti of Australian (Au) and Malaysian (My) backgrounds. Data from two replicate populations were pooled for visualization. Each point represents the relative density for an individual averaged across 2-3 technical replicates. Medians and 95\% confidence intervals are shown in black lines. 


\section{wAlbB shows complete maternal transmission regardless of nuclear background}

We observed complete maternal transmission of wAlbB in both the Australian and Malaysian nuclear backgrounds, with all 10 offspring from 10 females (100/100) in each population being infected (lower 95\% confidence interval: 0.96).

\section{wAlbB is stable under cyclical heat stress regardless of nuclear background}

To test the stability of $w A l b B$ at high temperatures in the different nuclear backgrounds, we measured Wolbachia densities in adults after eggs were exposed to cyclical heat stress $\left(29-39^{\circ} \mathrm{C}\right)$ or held at $26^{\circ} \mathrm{C}$. We found no significant effect of temperature (females: $F_{(1,56)}=2.073, P=0.155$, males: $F_{(1,56)}=0.676, P$ $=0.414$ ), nuclear background (females: $F_{(1,56)}=2.228, P=0.141$, males $\left(F_{(1,56)}=1.341, P=0.252\right)$, or interactions between temperature and nuclear background (females: $F_{(1,56)}=1.276, P=0.264$, males: $F_{(1}$, ${ }_{56}=0.749, P=0.391$ ), indicating that this infection is stable under heat stress regardless of nuclear background (Figure 6). In contrast, the wMel infection decreased in density by a median of $98.7 \%$ (across both sexes) under the same conditions (Figure 6). 


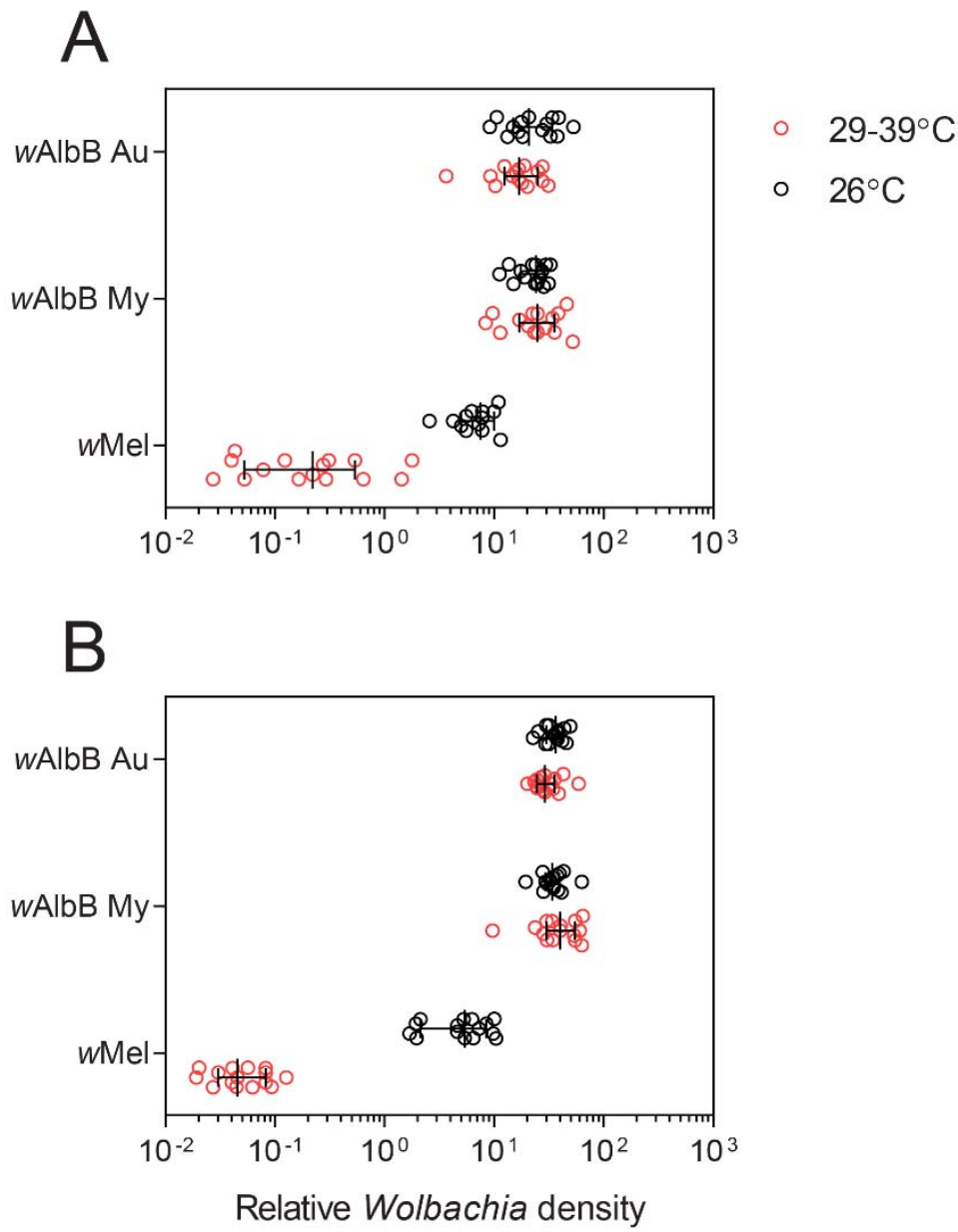

Figure 6. Wolbachia density in (A) females and (B) males following exposure to cyclical heat stress during the egg stage. Eggs from the wAlbB Au, wAlbB My and wMel populations were exposed to cyclical temperatures of $29-39^{\circ} \mathrm{C}$ for $7 \mathrm{~d}$ (red circles) or held at $26^{\circ} \mathrm{C}$ (black circles). Each point represents the relative density for an individual averaged across 2-3 technical replicates. Medians and $95 \%$ confidence intervals are shown in black lines.

wAlbB induces complete cytoplasmic incompatibility regardless of nuclear background and heat stress

We tested the ability of WAlbB-infected males to induce cytoplasmic incompatibility. Uninfected females that were crossed to WAlbB Au or wAlbB My males produced no viable offspring (no eggs hatched), indicating complete cytoplasmic incompatibility (Figure 7A). wAlbB-infected males continued to induce complete cytoplasmic incompatibility following exposure to cyclical temperatures of $29-39^{\circ} \mathrm{C}$ for $7 \mathrm{~d}$ during the egg stage, regardless of nuclear background. In contrast, heat stress weakened cytoplasmic incompatibility induction by wMel-infected males, with all uninfected females in this cross producing viable offspring (Figure 7A). 
We also tested the ability of wAlbB-infected females to restore compatibility with wAlbB-infected males under heat stress (Figure 7B). wAlbB-infected females exhibited high hatch proportions regardless of nuclear background or heat stress. Heat stress had no significant effect on the egg hatch proportions of wAlbB Au (Mann-Whitney $\mathrm{U}: \mathrm{Z}=1.512, \mathrm{P}=0.131$ ) or wAlbB My females $(\mathrm{Z}=0.585, \mathrm{P}=0.562)$, but greatly reduced the egg hatch of wMel-infected females $(Z=5.114, P<0.001)$. Overall, these results show that both cytoplasmic incompatibility induction and compatibility restoration are robust to nuclear background and heat treatment.
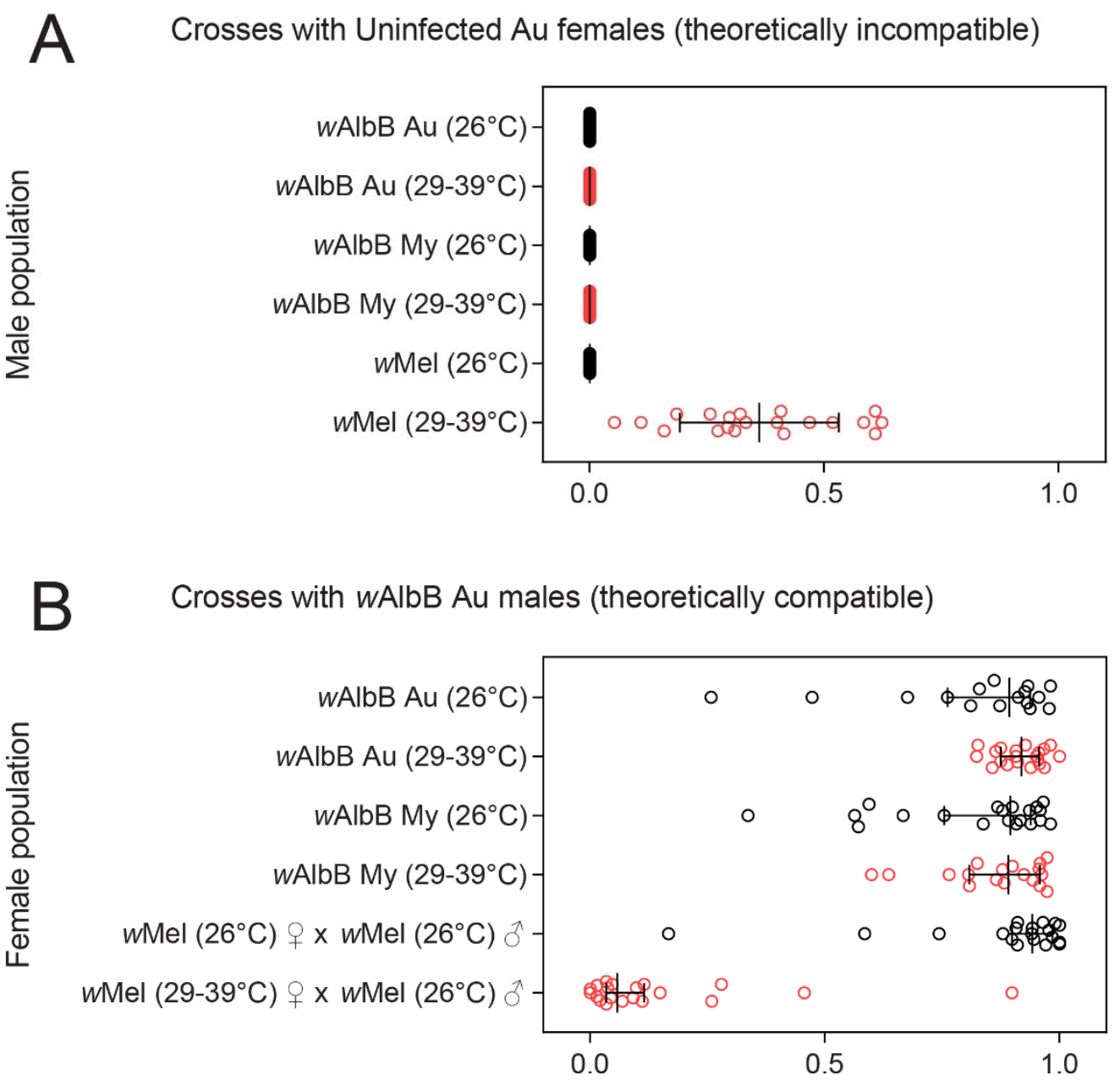

Egg hatch proportion

Figure 7. Cytoplasmic incompatibility induction and compatibility restoration following exposure to cyclical heat stress during the egg stage. We performed crosses to test the ability of $(A)$ Wolbachiainfected males to induce cytoplasmic incompatibility with uninfected females and (B) Wolbachiainfected females to restore compatibility with Wolbachia-infected males. In each cross, males (A) or females (B) from the wAlbB Au, wAlbB My and wMel populations were exposed to cyclical temperatures of $29-39^{\circ} \mathrm{C}$ for $7 \mathrm{~d}$ (red circles) or held at $26^{\circ} \mathrm{C}$ (black circles) during the egg stage. Each point represents 
the hatch proportion of eggs from a single female. Medians and $95 \%$ confidence intervals are shown in black lines.

\section{Discussion}

In this study, we generated a wAlbB transinfection $\left(\right.$ AAlbB $\left.^{Q}\right)$ in Ae. aegypti and evaluated its phenotypic effects in two nuclear backgrounds. wAlbB showed $100 \%$ maternal transmission, induced complete cytoplasmic incompatibility and was stable at high temperatures across both Australian and Malaysian backgrounds. Life history traits were driven largely by mosquito nuclear background, with Malaysian mosquitoes having larger wings and laying more eggs than Australian mosquitoes, but with lower quiescent egg viability. However, wAlbB infection induced substantial costs to egg hatch, particularly in quiescent eggs. These effects were consistent across two replicate populations. We found no clear deleterious effects of having mismatched mitochondrial and nuclear genomes, supporting the use of backcrossing as a method to introduce Wolbachia infections into target backgrounds for field release. Whole Wolbachia genome sequencing of the $\mathrm{WAlbB}^{\complement}$ transinfection revealed very few changes compared to the reference genome. Our results point to the stability of wAlbB infections across time, environment, and host background. The $\mathrm{WAlbB}^{\mathrm{Q}}$ transinfection generated in this study is therefore likely to retain desirable traits for arbovirus and mosquito control under a broad range of conditions.

WAlbB infections in Ae. aegypti typically induce host fitness costs (AXFORD et al. 2016; JOUBERT et al. 2016; ANT et al. 2018; LAU et al. 2021), but these effects may depend on both host and Wolbachia variation. CARVALHO et al. (2020) recently demonstrated the importance of host background, where wAlbB showed larger deleterious effects in a Mexican background compared to a Brazilian background. Here we found that $w A \mathrm{lbB}$ infection reduced egg hatch proportions and dramatically reduced quiescent egg viability, but effects were consistent across two mosquito backgrounds. Although the backgrounds used in CARVALHO et al. (2020) were different from ours, differences in outcomes could also be explained by methodological differences. We used antibiotic curing to ensure that mitochondrial haplotypes were matched between infected and uninfected lines, while CARVALHO et al. (2020) compared the wAlbBinfected populations to naturally-uninfected populations. However, we also used fewer rounds of backcrossing, resulting in incomplete introgression into the Malaysian background. Controlling for mitochondrial haplotype is an important consideration when elucidating Wolbachia phenotypes given that it can have substantial effects on host fitness in other insects (HOEKSTRA et al. 2013; MEIKLEJOHN et al. 2013; RANK et al. 2020).

We performed whole genome sequencing of our $w \mathrm{AlbB}^{\complement}$ transinfection to detect potential evolutionary changes and found that its sequence is almost identical to the reference genome (SINHA et al. 2019). The transinfection and reference genomes have a common origin; both wAlbB infections were derived from Ae. albopictus caught in Houston, Texas, USA in 1986 (SINKINS et al. 1995). However, their histories are distinct; the reference sequence was derived from a wAlbB infection maintained in the Ae. aegypti Aa23 cell line for more than two decades (O'NEILL et al. 1997), while the transinfection was introduced to Ae. 
aegypti over 15 years ago (XI et al. 2005b). The long-term stability of wAlbB is consistent with the wMel and wMelPop-CLA infections in Ae. aegypti, which also show few long-term genomic (WoolfiT et al. 2013; HUANG et al. 2020) and phenotypic (HOFFMANN et al. 2014; Ross et al. 2020a) changes following transinfection. These results suggest that Wolbachia infections will likely remain stable in their effects following field releases, broadly consistent with phenotypic data for a separate wAlbB transinfection following releases in Malaysia (AHMAD et al. 2021).

The wAlbB Wolbachia strain is widespread throughout natural populations of Ae. albopictus but is thought to exhibit low genetic diversity (BOURTZIS et al. 2014). Previous surveys have detected little or no genetic variation within wAlbB through a limited set of molecular markers (ARMBRUSTER et al. 2003; ALBUQUERQUE et al. 2011; DAs et al. 2014; Hu et al. 2020), but a recent reanalysis of Ae. albopictus genome sequence data points to substantial variation within this strain (SCHOLz et al. 2020). We compared the genome sequence of our $\mathrm{WAlbB}^{\mathrm{Q}}$ transinfection to two other publicly available sequences from Ae. albopictus caught in St. Augustine, Florida, USA in 2016 (KULKARNI et al. 2019a) and Haikou, Hainan, China in 2016 (KULKARNI et al. 2019b) and found that all three were distinct from each other. These results emphasize the need to reevaluate wAlbB diversity in Ae. albopictus, since variation within $w A l b B$ could influence control interventions targeting this species. This also raises the question of whether independently-derived wAlbB transinfections show phenotypic differences. For instance, the wAlbB transinfection generated by Xi et al. (2005b) is stable at high temperatures (Ross et al. 2017b) while the transinfection generated by Ant et al. (2018) that has been deployed in Malaysia (NAZNI et al. 2019) decreases in density under a similar temperature regime (ANT et al. 2018). Given the sequence variation within wAlbB, nomenclature for differentiating between variants (as for other Wolbachia strains such as WPip (ATYAME et al. 2011)) may be useful, particularly if they differ in their phenotypic effects.

Aedes albopictus is native to Asia and only invaded USA in the 1980s (BENEDICT et al. 2007). The initial incursion into mainland USA is thought to have originated in Japan, and to have been followed by a complex pattern of successive introductions from other locations (ZHONG et al. 2013; GOUBERT et al. 2016; KOTSAKIOZI et al. 2017; MANNI et al. 2017). Given that the Houston strain of Ae. albopictus, from which the $w A \mathrm{AlBB}^{\complement}$ transinfection and the reference variant are derived, was collected only one year after the first report of Ae. albopictus in mainland USA in 1985 (SPRENGER AND WUITHIRANYAGOOL 1986), it is likely that this strain originated in Japan. Recent population genetic studies of Ae. albopictus have identified clear patterns of geographic differentiation among populations within the native Asian range of the species (KOTSAKIOZI et al. 2017; SCHMIDT et al. 2020). Consistent with this, our phylogenomic analysis separated the wAlbB variants into three main branches: a Chinese branch, a Florida Branch (of unknown origin) and a probable Japanese branch. Understanding the extent of variation within wAlbB in natural populations could provide insights into the speciation and global spread of Ae. albopictus.

In conclusion, we have shown that phenotypic effects of the wAlbB infection tested here (and associated mtDNA) are stable across nuclear backgrounds, providing little evidence for nuclear genome-Wolbachia interactions or changes in wAlbB associated with multiple host transfers through microinjection. Our results have implications for releases of Wolbachia-infected Ae. aegypti that are taking place in different 
mosquito backgrounds around the world. They suggest that one source of infection may serve releases in multiple locations, although adequate backcrossing remains important to ensure that the nuclear background is consistent with that of target populations for genes under local selection such as those involved with pesticide resistance (ENDERSBY-HARSHMAN et al. 2020).

\section{Acknowledgements}

We thank Meng-Jia Lau, Véronique Paris, Moshe Jasper, Ashley Callahan, Jason Axford and Vanessa White for technical assistance and lan Forster and Trent Perry for needle pulling assistance. We thank Zhiyong Xi for providing the original wAlbB transinfection used in this study.

\section{Funding}

AAH was supported by the National Health and Medical Research Council (1132412, 1118640, www.nhmrc.gov.au). PAR was supported by a University of Melbourne Early Career Research Grant. The funders had no role in study design, data collection and analysis, decision to publish, or preparation of the manuscript.

\section{Data availability}

The whole genome sequence of the wAlbB $^{Q}$ transinfection will be deposited in Genbank following submission.

\section{Supplementary information}

Table S1. Generation of a stable wAlbB transinfection ( $\left(\mathrm{AlbB}^{\mathrm{a}}\right.$ ) in an Australian mitochondrial and nuclear background of Aedes aegypti through embryonic microinjection.

\begin{tabular}{|l|l|l|l|l|l|l|l|}
\hline Technique & $\begin{array}{l}\text { Eggs } \\
\text { injected }\end{array}$ & $\begin{array}{l}\text { Male } \mathrm{F}_{0} \\
\text { adults } \\
\text { (wAlbB- } \\
\text { positive) }\end{array}$ & $\begin{array}{l}\text { Female } \\
\mathrm{F}_{0} \\
\text { adults } \\
\text { (wAlbB- } \\
\text { positive) }\end{array}$ & $\begin{array}{l}\text { Female } \\
\mathrm{F}_{1} \\
\text { adults } \\
\text { tested } \\
\text { (wAlbB- } \\
\text { positive) }\end{array}$ & $\begin{array}{l}\text { Female } \\
\mathrm{F}_{2} \\
\text { adults } \\
\text { tested } \\
\text { (wAlbB- } \\
\text { positive) }\end{array}$ & $\begin{array}{l}\text { Female } \\
\mathrm{F}_{3} \\
\text { adults } \\
\text { tested } \\
\text { (wAlbB- } \\
\text { positive) }\end{array}$ & $\begin{array}{l}\text { Female } \\
\mathrm{F}_{4} \\
\text { adults } \\
\text { tested } \\
\text { (wAlbB- } \\
\text { positive) }\end{array}$ \\
\hline $\begin{array}{l}\text { Ovary } \\
\text { homogenate }\end{array}$ & 1165 & $31(1)$ & $31(3)$ & $60(0)$ & - & - & - \\
\hline Cytoplasm & 396 & $20(5)$ & $22(3)$ & $57(1)$ & $23(19)$ & $39(39)$ & $54(54)$ \\
\hline
\end{tabular}


Table S2. Haplotype combinations for the mitochondrial COI region.

\begin{tabular}{|c|c|c|c|c|c|c|c|c|c|c|c|c|}
\hline \multirow[t]{2}{*}{ Mitochondrial haplotype } & \multicolumn{12}{|c|}{ SNP position (750 bp fragment) } \\
\hline & 3 & 84 & 192 & 414 & 426 & 531 & 579 & 594 & 630 & 636 & 682 & 744 \\
\hline 1 & $\mathrm{C}$ & $\mathrm{T}$ & $\mathrm{C}$ & $T$ & $T$ & A & $\mathrm{C}$ & A & $\mathrm{C}$ & $\mathrm{T}$ & $\mathrm{T}$ & $T$ \\
\hline 2 & $\mathrm{~T}$ & $\mathrm{C}$ & $\mathrm{C}$ & $\mathrm{C}$ & C & $\mathrm{G}$ & $\mathrm{T}$ & G & $\mathrm{T}$ & $\mathrm{C}$ & $\mathrm{C}$ & $T$ \\
\hline 3 & $\mathrm{C}$ & $\mathrm{T}$ & C & $\mathrm{T}$ & $T$ & G & C & A & C & $\mathrm{T}$ & $\mathrm{T}$ & $T$ \\
\hline 4 & $\mathrm{~T}$ & $\mathrm{~T}$ & $\mathrm{~T}$ & $\mathrm{C}$ & C & G & $\mathrm{T}$ & $\mathrm{G}$ & $\mathrm{T}$ & $\mathrm{T}$ & $C$ & $\mathrm{~T}$ \\
\hline 5 & $\mathrm{C}$ & $\mathrm{T}$ & $\mathrm{C}$ & $\bar{T}$ & $T$ & $\mathrm{G}$ & $\mathrm{C}$ & $A$ & $\mathrm{C}$ & $\bar{T}$ & $\bar{T}$ & $\mathrm{G}$ \\
\hline
\end{tabular}

Appendix S1. Datasets used for phylogenomic analyses.

Appendix S2. Protein accessions for each orthogroup.

Appendix S3. Genomic analysis of wAlbB variant polymorphisms.

\section{References}

Ahmad, N. A., M. V. Mancini, T. H. Ant, J. Martinez, G. M. R. Kamarul et al., 2021 Wolbachia strain wAlbB maintains high density and dengue inhibition following introduction into a field population of Aedes aegypti. Philos Trans R Soc Lond B Biol Sci 376: 20190809.

Albuquerque, A. L. d., T. Magalhães and C. F. J. Ayres, 2011 High prevalence and lack of diversity of Wolbachia pipientis in Aedes albopictus populations from Northeast Brazil. Mem Inst Oswaldo Cruz 106: 773-776.

Ant, T. H., C. S. Herd, V. Geoghegan, A. A. Hoffmann and S. P. Sinkins, 2018 The Wolbachia strain wAu provides highly efficient virus transmission blocking in Aedes aegypti. PLoS Pathog 14: e1006815.

Armbruster, P., W. E. Damsky, R. Giordano, J. Birungi, L. E. Munstermann et al., 2003 Infection of Newand Old-World Aedes albopictus (Diptera: Culicidae) by the intracellular parasite Wolbachia: Implications for host mitochondrial DNA evolution. J Med Ent 40: 356-360.

Atyame, C. M., F. Delsuc, N. Pasteur, M. Weill and O. Duron, 2011 Diversification of Wolbachia endosymbiont in the Culex pipiens mosquito. Mol Biol Evol 28: 2761-2772.

Axford, J. K., P. A. Ross, H. L. Yeap, A. G. Callahan and A. A. Hoffmann, 2016 Fitness of wAlbB Wolbachia infection in Aedes aegypti: parameter estimates in an outcrossed background and potential for population invasion. Am J Trop Med Hyg 94: 507-516.

Benedict, M. Q., R. S. Levine, W. A. Hawley and L. P. Lounibos, 2007 Spread of the tiger: global risk of invasion by the mosquito Aedes albopictus. Vector Borne Zoonotic Dis 7: 76-85.

Bhattacharya, T., I. L. Newton and R. W. Hardy, 2020 Viral RNA is a target for Wolbachia-mediated pathogen blocking. PLoS Pathog 16: e1008513. 
Bian, G., Y. Xu, P. Lu, Y. Xie and Z. Xi, 2010 The endosymbiotic bacterium Wolbachia induces resistance to dengue virus in Aedes aegypti. PLoS Pathog 6: e1000833.

Bolger, A. M., M. Lohse and B. Usadel, 2014 Trimmomatic: a flexible trimmer for Illumina sequence data. Bioinformatics 30: 2114-2120.

Bordenstein, S. R., J. J. Uy and J. H. Werren, 2003 Host genotype determines cytoplasmic incompatibility type in the haplodiploid genus Nasonia. Genetics 164: 223-233.

Bourtzis, K., S. L. Dobson, Z. Xi, J. L. Rasgon, M. Calvitti et al., 2014 Harnessing mosquito-Wolbachia symbiosis for vector and disease control. Acta Tropica 132 Suppl: S150-163.

Carvalho, D. O., J. A. Torres-Monzon, P. Koskinioti, N. D. A. Dilrukshi Wijegunawardana, X. Liang et al., 2020 Aedes aegypti lines for combined sterile insect technique and incompatible insect technique applications: the importance of host genomic background. Entomol Exp Appl 168: 560-572.

Castresana, J., 2000 Selection of conserved blocks from multiple alignments for their use in phylogenetic analysis. Mol Biol Evol 17: 540-552.

Crawford, J. E., D. W. Clarke, V. Criswell, M. Desnoyer, D. Cornel et al., 2020 Efficient production of male Wolbachia-infected Aedes aegypti mosquitoes enables large-scale suppression of wild populations. Nat Biotechnol 38: 482-492.

Danecek, P., J. K. Bonfield, J. Liddle, J. Marshall, V. Ohan et al., 2021 Twelve years of SAMtools and BCFtools. GigaScience 10: giab008.

Das, B., T. Satapathy, S. K. Kar and R. K. Hazra, 2014 Genetic structure and Wolbachia genotyping in naturally occurring populations of Aedes albopictus across contiguous landscapes of Orissa, India. PLoS One 9: e94094.

Dean, M. D., 2006 A Wolbachia-associated fitness benefit depends on genetic background in Drosophila simulans. Proc R Soc Lond B Biol Sci 273: 1415-1420.

Dutra, H. L., L. M. Dos Santos, E. P. Caragata, J. B. Silva, D. A. Villela et al., 2015 From lab to field: The influence of urban landscapes on the invasive potential of Wolbachia in Brazilian Aedes aegypti mosquitoes. PLoS NegI Trop Dis 9: e0003689.

Emms, D. M., and S. Kelly, 2019 OrthoFinder: phylogenetic orthology inference for comparative genomics. Genome Biol 20: 1-14.

Endersby-Harshman, N. M., J. K. Axford and A. A. Hoffmann, 2019 Environmental concentrations of antibiotics may diminish Wolbachia infections in Aedes aegypti (Diptera: Culicidae). J Med Ent 56: 1078-1086.

Endersby-Harshman, N. M., T. L. Schmidt, J. Chung, A. van Rooyen, A. R. Weeks et al., 2020 Heterogeneous genetic invasions of three insecticide resistance mutations in Indo-Pacific populations of Aedes aegypti (L.). Mol Ecol 29: 1628-1641.

Flores, H. A., J. Taneja de Bruyne, T. B. O'Donnell, V. Tuyet Nhu, N. Thi Giang et al., 2020 Multiple Wolbachia strains provide comparative levels of protection against dengue virus infection in Aedes aegypti. PLoS Pathog 16: e1008433.

Foo, I. J.-H., A. A. Hoffmann and P. A. Ross, 2019 Cross-generational effects of heat stress on fitness and Wolbachia density in Aedes aegypti mosquitoes. Trop Med Infect Dis 4: 13.

Ford, S. A., S. L. Allen, J. R. Ohm, L. T. Sigle, A. Sebastian et al., 2019 Selection on Aedes aegypti alters Wolbachia-mediated dengue virus blocking and fitness. Nat Microbiol 4: 1832-1839.

Fraser, J. E., J. T. De Bruyne, I. Iturbe-Ormaetxe, J. Stepnell, R. L. Burns et al., 2017 Novel Wolbachiatransinfected Aedes aegypti mosquitoes possess diverse fitness and vector competence phenotypes. PLoS Pathog 13: e1006751.

Garcia, G. d. A., G. Sylvestre, R. Aguiar, G. B. da Costa, A. J. Martins et al., 2019 Matching the genetics of released and local Aedes aegypti populations is critical to assure Wolbachia invasion. PLoS Negl Trop Dis 13: e0007023. 
Gesto, J. S. M., S. Pinto, F. B. S. Dias, J. Peixoto, G. Costa et al., 2021 Large-scale deployment and establishment of Wolbachia into the Aedes aegypti population in Rio de Janeiro, Brazil. bioRxiv: 2021.2004.2029.441982.

Gloria-Soria, A., D. Ayala, A. Bheecarry, O. Calderon-Arguedas, D. D. Chadee et al., 2016 Global genetic diversity of Aedes aegypti. Mol Ecol 25: 5377-5395.

Gloria-Soria, A., T. G. Chiodo and J. R. Powell, 2018 Lack of evidence for natural Wolbachia infections in Aedes aegypti (Diptera: Culicidae). J Med Entomol 55: 1354-1356.

Goubert, C., G. Minard, C. Vieira and M. Boulesteix, 2016 Population genetics of the Asian tiger mosquito Aedes albopictus, an invasive vector of human diseases. Heredity 117: 125-134.

Hoekstra, L. A., M. A. Siddiq and K. L. Montooth, 2013 Pleiotropic effects of a mitochondrial-nuclear incompatibility depend upon the accelerating effect of temperature in Drosophila. Genetics 195: 1129-1139.

Hoffmann, A. A., I. Iturbe-Ormaetxe, A. G. Callahan, B. L. Phillips, K. Billington et al., 2014 Stability of the wMel Wolbachia infection following invasion into Aedes aegypti populations. PLoS Negl Trop Dis 8: e3115.

Hoffmann, A. A., B. L. Montgomery, J. Popovici, I. Iturbe-Ormaetxe, P. H. Johnson et al., 2011 Successful establishment of Wolbachia in Aedes populations to suppress dengue transmission. Nature 476: 454-457.

Hu, Y., Z. Xi, X. Liu, J. Wang, Y. Guo et al., 2020 Identification and molecular characterization of Wolbachia strains in natural populations of Aedes albopictus in China. Parasit Vectors 13: 28.

Huang, B., Q. Yang, A. A. Hoffmann, S. A. Ritchie, A. F. van den Hurk et al., 2020 Wolbachia genome stability and mtDNA variants in Aedes aegypti field populations eight years after release. iScience 23: 101572.

Indriani, C., W. Tantowijoyo, E. Rancès, B. Andari, E. Prabowo et al., 2020 Reduced dengue incidence following deployments of Wolbachia-infected Aedes aegypti in Yogyakarta, Indonesia: A quasiexperimental trial using controlled interrupted time series analysis. Gates Open Res 4.

Jasper, M. E., Q. Yang, P. A. Ross, N. Endersby-Harshman, N. Bell et al., 2019 A LAMP assay for the rapid and robust assessment of Wolbachia infection in Aedes aegypti under field and laboratory conditions. PLoS One 14: e0225321.

Joubert, D. A., T. Walker, L. B. Carrington, J. T. De Bruyne, D. H. Kien et al., 2016 Establishment of a Wolbachia superinfection in Aedes aegypti mosquitoes as a potential approach for future resistance management. PLoS Pathog 12: e1005434.

Katoh, K., and D. M. Standley, 2013 MAFFT multiple sequence alignment software version 7: improvements in performance and usability. Mol Biol Evol 30: 772-780.

Kong, J. D., J. K. Axford, A. A. Hoffmann and M. R. Kearney, 2016 Novel applications of thermocyclers for phenotyping invertebrate thermal responses. Methods Ecol Evol 7: 1201-1208.

Kotsakiozi, P., J. B. Richardson, V. Pichler, G. Favia, A. J. Martins et al., 2017 Population genomics of the Asian tiger mosquito, Aedes albopictus: insights into the recent worldwide invasion. Ecol Evol 7: 10143-10157.

Kulkarni, A., W. Yu, R.-D. Xue, Y. Ma and J. Xu, 2019a Nucleotide [Internet]. Bethesda (MD): National Library of Medicine (US), National Center for Biotechnology Information; [1988] - . Accession No. CP041923.1, Wolbachia pipientis strain wAlbB-FL2016 chromosome, complete genome. Available from: https://www.ncbi.nlm.nih.gov/nuccore/CP041923, pp.

Kulkarni, A., W. Yu, R.-D. Xue, Y. Ma and J. Xu, 2019b Nucleotide [Internet]. Bethesda (MD): National Library of Medicine (US), National Center for Biotechnology Information; [1988] - . Accession No. CP041924.1, Wolbachia pipientis strain WAlbB-HN2016 chromosome, complete genome. Available from: https://www.ncbi.nlm.nih.gov/nuccore/CP041924. 
Kyritsis, G. A., A. A. Augustinos, I. Livadaras, C. Caceres, K. Bourtzis et al., 2019 Medfly-Wolbachia symbiosis: genotype $\mathrm{x}$ genotype interactions determine host's life history traits under mass rearing conditions. BMC Biotechnol 19: 96.

Lau, M.-J., P. A. Ross and A. A. Hoffmann, 2021 Infertility and fecundity loss of Wolbachia-infected Aedes aegypti hatched from quiescent eggs is expected to alter invasion dynamics. PLoS Negl Trop Dis 15: e0009179.

Lee, S. F., V. L. White, A. R. Weeks, A. A. Hoffmann and N. M. Endersby, 2012 High-throughput PCR assays to monitor Wolbachia infection in the dengue mosquito (Aedes aegypti) and Drosophila simulans. Appl Environ Microbiol 78: 4740-4743.

Li, H., 2013 Aligning sequence reads, clone sequences and assembly contigs with BWA-MEM. arXiv: 1303.3997.

Li, H., B. Handsaker, A. Wysoker, T. Fennell, J. Ruan et al., 2009 The sequence alignment/map format and SAMtools. Bioinformatics 25: 2078-2079.

Lu, P., G. Bian, X. Pan and Z. Xi, 2012 Wolbachia induces density-dependent inhibition to dengue virus in mosquito cells. PLoS Negl Trop Dis 6: e1754.

Lunt, D., D. X. Zhang, J. Szymura and O. Hewltt, 1996 The insect cytochrome oxidase I gene: evolutionary patterns and conserved primers for phylogenetic studies. Insect Mol Biol 5: 153-165.

Mains, J. W., P. H. Kelly, K. L. Dobson, W. D. Petrie and S. L. Dobson, 2019 Localized control of Aedes aegypti (Diptera: Culicidae) in Miami, FL, via inundative releases of Wolbachia-infected male mosquitoes. J Med Ent 56: 1296-1303.

Manni, M., C. R. Guglielmino, F. Scolari, A. Vega-Rua, A. B. Failloux et al., 2017 Genetic evidence for a worldwide chaotic dispersion pattern of the arbovirus vector, Aedes albopictus. PLoS Negl Trop Dis 11: e0005332.

McMeniman, C. J., R. V. Lane, B. N. Cass, A. W. Fong, M. Sidhu et al., 2009 Stable introduction of a lifeshortening Wolbachia infection into the mosquito Aedes aegypti. Science 323: 141-144.

Meiklejohn, C. D., M. A. Holmbeck, M. A. Siddiq, D. N. Abt, D. M. Rand et al., 2013 An incompatibility between a mitochondrial tRNA and its nuclear-encoded tRNA synthetase compromises development and fitness in Drosophila. PLoS Genet 9: e1003238.

Miller, M. A., W. Pfeiffer and T. Schwarz, 2010 Creating the CIPRES Science Gateway for inference of large phylogenetic trees, pp. 1-8 in Proceedings of the Gateway Computing Environments Workshop (GCE), New Orleans, LA.

Mousson, L., K. Zouache, C. Arias-Goeta, V. Raquin, P. Mavingui et al., 2012 The native Wolbachia symbionts limit transmission of dengue virus in Aedes albopictus. PLoS Negl Trop Dis 6: e1989.

Nazni, W. A., A. A. Hoffmann, A. NoorAfizah, Y. L. Cheong, M. V. Mancini et al., 2019 Establishment of Wolbachia strain wAlbB in Malaysian populations of Aedes aegypti for dengue control. Curr Biol 29: 4241-4248 e4245.

O'Neill, S. L., M. M. Pettigrew, S. Sinkins, H. Braig, T. Andreadis et al., 1997 In vitro cultivation of Wolbachia pipientis in an Aedes albopictus cell line. Insect Mol Biol 6: 33-39.

O'Neill, S. L., P. A. Ryan, A. P. Turley, G. Wilson, K. Retzki et al., 2018 Scaled deployment of Wolbachia to protect the community from dengue and other Aedes transmitted arboviruses. Gates Open Res 2.

Paris, V., E. Cottingham, P. A. Ross, J. K. Axford and A. A. Hoffmann, 2018 Effects of alternative blood sources on Wolbachia infected Aedes aegypti females within and across generations. Insects 9: 140.

Pinto, S. B., T. I. Riback, G. Sylvestre, G. Costa, J. Peixoto et al., 2021 Effectiveness of Wolbachia-infected mosquito deployments in reducing the incidence of dengue and other Aedes-borne diseases in Niterói, Brazil: a quasi-experimental study. medRxiv: 2021.2001.2031.21250726.

Ramírez, A. L., 2018 Mosquitoes. figshare: https://doi.org/10.6084/m6089.figshare.c.4208051. 
Rank, N. E., P. Mardulyn, S. J. Heidl, K. T. Roberts, N. A. Zavala et al., 2020 Mitonuclear mismatch alters performance and reproductive success in naturally introgressed populations of a montane leaf beetle. Evolution 74: 1724-1740.

Rasgon, J. L., A. J. Cornel and T. W. Scott, 2006 Evolutionary history of a mosquito endosymbiont revealed through mitochondrial hitchhiking. Proceedings of the Royal Society B: Biological Sciences 273: 1603-1611.

Reynolds, K. T., and A. A. Hoffmann, 2002 Male age, host effects and the weak expression or nonexpression of cytoplasmic incompatibility in Drosophila strains infected by maternally transmitted Wolbachia. Genet Res 80: 79-87.

Reynolds, K. T., L. J. Thomson and A. A. Hoffmann, 2003 The effects of host age, host nuclear background and temperature on phenotypic effects of the virulent Wolbachia strain popcorn in Drosophila melanogaster. Genetics 164: 1027-1034.

Ritchie, S. A., M. Townsend, C. J. Paton, A. G. Callahan and A. A. Hoffmann, 2015 Application of wMelPop Wolbachia strain to crash local populations of Aedes aegypti. PLoS Negl Trop Dis 9: e0003930.

Ross, P. A., J. K. Axford, A. G. Callahan, K. M. Richardson and A. A. Hoffmann, 2020a Persistent deleterious effects of a deleterious Wolbachia infection. PLoS Negl Trop Dis 14: e0008204.

Ross, P. A., J. K. Axford, K. M. Richardson, N. M. Endersby-Harshman and A. A. Hoffmann, 2017a Maintaining Aedes aegypti mosquitoes infected with Wolbachia. J Vis Exp: e56124.

Ross, P. A., A. G. Callahan, Q. Yang, M. Jasper, M. A. K. Arif et al., 2020b An elusive endosymbiont: Does Wolbachia occur naturally in Aedes aegypti? Ecol Evol 10: 1581-1591.

Ross, P. A., N. M. Endersby-Harshman and A. A. Hoffmann, 2019a A comprehensive assessment of inbreeding and laboratory adaptation in Aedes aegypti mosquitoes. Evol Appl 12: 572-586.

Ross, P. A., N. M. Endersby and A. A. Hoffmann, 2016 Costs of three Wolbachia infections on the survival of Aedes aegypti larvae under starvation conditions. PLoS Negl Trop Dis 10: e0004320.

Ross, P. A., S. A. Ritchie, J. K. Axford and A. A. Hoffmann, 2019b Loss of cytoplasmic incompatibility in Wolbachia-infected Aedes aegypti under field conditions. PLoS Negl Trop Dis 13: e0007357.

Ross, P. A., M. Turelli and A. A. Hoffmann, 2019c Evolutionary ecology of Wolbachia releases for disease control. Annu Rev Genet 53: 93-116.

Ross, P. A., I. Wiwatanaratanabutr, J. K. Axford, V. L. White, N. M. Endersby-Harshman et al., 2017b Wolbachia infections in Aedes aegypti differ markedly in their response to cyclical heat stress. PLoS Pathog 13: e1006006.

Ryan, P. A., A. P. Turley, G. Wilson, T. P. Hurst, K. Retzki et al., 2019 Establishment of wMel Wolbachia in Aedes aegypti mosquitoes and reduction of local dengue transmission in Cairns and surrounding locations in northern Queensland, Australia. Gates Open Research 3.

Sazama, E. J., S. P. Ouellette and J. S. Wesner, 2019 Bacterial endosymbionts are common among, but not necessarily within, insect species. Environ Entomol 48: 127-133.

Schmidt, T. L., N. H. Barton, G. Rasic, A. P. Turley, B. L. Montgomery et al., 2017 Local introduction and heterogeneous spatial spread of dengue-suppressing Wolbachia through an urban population of Aedes aegypti. PLoS Biol 15: e2001894.

Schmidt, T. L., J. Chung, A.-C. Honnen, A. R. Weeks and A. A. Hoffmann, 2020 Population genomics of two invasive mosquitoes (Aedes aegypti and Aedes albopictus) from the Indo-Pacific. PLoS neglected tropical diseases 14: e0008463.

Scholz, M., D. Albanese, K. Tuohy, C. Donati, N. Segata et al., 2020 Large scale genome reconstructions illuminate Wolbachia evolution. Nat Commun 11: 5235.

Sinha, A., Z. Li, L. Sun and C. K. S. Carlow, 2019 Complete genome sequence of the Wolbachia wAlbB endosymbiont of Aedes albopictus. Genome Biol Evol 11: 706-720.

Sinkins, S. P., H. R. Braig and S. L. O'Neill, 1995 Wolbachia superinfections and the expression of cytoplasmic incompatibility. Proc Biol Sci 261: 325-330. 
Sprenger, D., and T. Wuithiranyagool, 1986 The discovery and distribution of Aedes albopictus in Harris County, Texas. J Am Mosq Control Assoc 2: 217-219.

Stamatakis, A., 2014 RAxML version 8: a tool for phylogenetic analysis and post-analysis of large phylogenies. Bioinformatics 30: 1312-1313.

Tantowijoyo, W., B. Andari, E. Arguni, N. Budiwati, I. Nurhayati et al., 2020 Stable establishment of wMel Wolbachia in Aedes aegypti populations in Yogyakarta, Indonesia. PLoS Negl Trop Dis 14: e0008157.

Terradas, G., S. L. Allen, S. F. Chenoweth and E. A. McGraw, 2017 Family level variation in Wolbachiamediated dengue virus blocking in Aedes aegypti. Parasit Vectors 10: 622.

Terradas, G., and E. A. McGraw, 2017 Wolbachia-mediated virus blocking in the mosquito vector Aedes aegypti. Curr Opin Insect Sci 22: 37-44.

Walker, T., P. H. Johnson, L. A. Moreira, I. Iturbe-Ormaetxe, F. D. Frentiu et al., 2011 The wMel Wolbachia strain blocks dengue and invades caged Aedes aegypti populations. Nature 476: 450453.

Weinert, L. A., E. V. Araujo-Jnr, M. Z. Ahmed and J. J. Welch, 2015 The incidence of bacterial endosymbionts in terrestrial arthropods. Proc Biol Sci 282: 20150249.

Woolfit, M., I. Iturbe-Ormaetxe, J. C. Brownlie, T. Walker, M. Riegler et al., 2013 Genomic evolution of the pathogenic Wolbachia strain, wMelPop. Genome Biol Evol 5: 2189-2204.

Xi, Z., J. L. Dean, C. Khoo and S. L. Dobson, 2005a Generation of a novel Wolbachia infection in Aedes albopictus (Asian tiger mosquito) via embryonic microinjection. Insect Biochem Mol Biol 35: 903910.

Xi, Z., and S. L. Dobson, 2005 Characterization of Wolbachia transfection efficiency by using microinjection of embryonic cytoplasm and embryo homogenate. Appl Environ Microbiol 71: 3199-3204.

Xi, Z., C. C. Khoo and S. L. Dobson, 2005b Wolbachia establishment and invasion in an Aedes aegypti laboratory population. Science 310: 326-328.

Yeap, H. L., G. Rasic, N. M. Endersby-Harshman, S. F. Lee, E. Arguni et al., 2016 Mitochondrial DNA variants help monitor the dynamics of Wolbachia invasion into host populations. Heredity (Edinb) 116: 265-276.

Zheng, X., D. Zhang, Y. Li, C. Yang, Y. Wu et al., 2019 Incompatible and sterile insect techniques combined eliminate mosquitoes. Nature 572: 56-61.

Zhong, D., E. Lo, R. Hu, M. E. Metzger, R. Cummings et al., 2013 Genetic analysis of invasive Aedes albopictus populations in Los Angeles County, California and its potential public health impact. PloS one 8: e68586. 